\title{
Immunohistochemical evaluation of D2-40, Galectin-3, Maspin and MCM7 expression in palate squamous cell carcinomas
}

\author{
AdRIAN PĂTRU ${ }^{1,2)}$, VAlERIU ŞURLIN ${ }^{1,3)}$, CLAUdiU MăRgĂRITESCU4), EdUARD MiHAi CiUCĂ2), \\ MARIUS MATEI ${ }^{5)}$, DANIELA DUMITRESCU ${ }^{6}$, , AdRIAN CAMEN ${ }^{2)}$ \\ ${ }^{1)}$ Doctoral School, University of Medicine and Pharmacy of Craiova, Romania \\ ${ }^{2)}$ Department of Oral and Maxillofacial Surgery, University of Medicine and Pharmacy of Craiova, Romania \\ ${ }^{3)}$ Department of Surgery, University of Medicine and Pharmacy of Craiova, Romania \\ 4) Department of Pathology, University of Medicine and Pharmacy of Craiova, Romania \\ 5) Department of Histology, University of Medicine and Pharmacy of Craiova, Romania \\ 6) Department of Radiology and Medical Imaging, University of Medicine and Pharmacy of Craiova, Romania
}

\begin{abstract}
Squamous cell carcinoma (SCC) is the most frequent cancer in oral cavity and its prognosis has exhibited little improvement in the last decades. Although much less common palate SCCs manifests a higher local aggression invading very quickly the adjacent muscles and jawbones, thus being able frequently to lead to dysfunctions in chewing, swallowing, and speech. To elucidate what underlies such local aggression, we investigated the immunohistochemical expression in palate SCCs of Podoplanin (D2-40), Galectin-3 (Gal-3), mammary serine protease inhibitor (Maspin) and minichromosome maintenance complex component 7 (MCM7), markers that are known to be involved in tumor invasiveness. We found a progressive increase in reactivity for D2-40 and MCM7 from the normal epithelium toward dysplastic epithelium and respectively to SCC, which suggests the intervention of these markers in the early stages of squamous cell carcinogenesis in the palate. The highest D2-40, Gal-3 and MCM7 reactivity was observed in basaloid and in poorly differentiated (G3) palate SCCs, while for Maspin the well-differentiated (G1) palate SCCs were the most reactive. The first three markers mentioned above were most intensely expressed at the invasion front, while the Maspin reactivity was low or absent at this level. Statistically, we found significant stratification on localization, grading, muscle invasion, and survival for all investigated markers, but with very high direct correlations between D2-40, Gal-3, and MCM7 immunoreactive score (IRS) values, while between the Maspin and each of the previous markers there were very high inverse correlations. Overall, all these investigate markers proved to be responsible for the local invasiveness and regional lymph node metastasis, thus allowing a prognostic and therapeutic stratification of patients with palate SCCs.
\end{abstract}

Keywords: palate, squamous cell carcinomas, D2-40, Galectin-3, Maspin, MCM7.

\section{a Introduction}

Squamous cell carcinoma (SCC) is the most frequent cancer of the oral cavity [1] and its prognosis has exhibited little improvement in the last decades [2]. It arises anywhere in the oral cavity, and tongue is the most common subsite being associated with higher mortality than other oral locations of SCC [3]. It is well known that oral SCC is prone to invade the deeper muscles and jawbones, reason for which the tumor depth of the invasion was included in tumor (T) stage of $8^{\text {th }}$ edition of the Union for International Cancer Control (UICC) and American Joint Committee on Cancer (AJCC)8 Staging Manual [4]. Although much less common palate SCCs manifests the same local aggression and frequently may causes dysfunctions in chewing, swallowing, and speech [5].

\section{Aim}

To elucidate what underlies such local aggression, we investigated the immunohistochemical (IHC) expression in palate SCCs of Podoplanin (D2-40), Galectin-3 (Gal-3), mammary serine protease inhibitor (Maspin) and minichromosome maintenance complex component 7 (MCM7), markers that are known to be involved in tumor invasiveness.

\section{Materials and Methods}

A retrospective study between 2010-2019 was conducted investigating patients diagnosed with palate SCC in the Department of Oral and Maxillofacial Surgery, Emergency County Hospital, Craiova, Romania. Reviewing the medical records, 45 cases of palate SCC were selected. Their main clinical and histopathological (HP) data were specified in a previous article [6]. As material for our study, we used the corresponding paraffin blocks of the included patients. From these, $4 \mu \mathrm{m}$-thick seriate sections were cut, which then were deparaffinized, hydrated and processed for immunohistochemistry according to a protocol detailed in a previous article [7]. The primary antibodies together with their characteristics are specified in the Table 1. For

This is an open-access article distributed under the terms of a Creative Commons Attribution-NonCommercial-ShareAlike 4.0 International Public License, which permits unrestricted use, adaptation, distribution and reproduction in any medium, non-commercially, provided the new creations are licensed under identical terms as the original work and the original work is properly cited. 
antigen unmasking, the slides were incubated with $0.1 \mathrm{M}$ pH 6 citrate buffer for Maspin and D2-40, and with TrisEthylenediaminetetraacetic acid (EDTA) buffer, $\mathrm{pH} 9.0$ for Gal-3 and MCM-7, and microwaved at $650 \mathrm{~W}$ for 20 minutes, and then cooled at room temperature for further 30 minutes.

Table 1 - The antibodies and immunostaining protocol

\begin{tabular}{cccc}
\hline Antibody & $\begin{array}{c}\text { Clone / Manufacturer, } \\
\text { Catalog No. }\end{array}$ & Dilution & $\begin{array}{c}\text { External } \\
\text { positive } \\
\text { control }\end{array}$ \\
\hline Podoplanin & $\begin{array}{c}\text { Mouse, monoclonal D2-40 / } \\
\text { Agilent, M3619 }\end{array}$ & $1: 100$ & Mesothelioma \\
\hline Gal-3 & $\begin{array}{c}\text { Mouse, monoclonal / } \\
\text { Novocastra, NCL-GAL-3 }\end{array}$ & $1: 200$ & $\begin{array}{c}\text { Colon } \\
\text { carcinoma }\end{array}$ \\
\hline Maspin & $\begin{array}{c}\text { Rabbit, polyclonal / } \\
\text { LifeSpan BioSciences, } \\
\text { LS-C352659 }\end{array}$ & $1: 100$ & $\begin{array}{c}\text { Breast } \\
\text { carcinoma }\end{array}$ \\
\hline MCM7 & $\begin{array}{c}\text { Rabbit, polyclonal / } \\
\text { Thermo Scientific, } \\
\text { PA5-82122 }\end{array}$ & $1: 300$ & Appendix \\
\hline
\end{tabular}

Gal-3: Galectin-3; Maspin: Mammary serine protease inhibitor; MCM7 Minichromosome maintenance complex component 7.

The IHC assessment, respective the immunoreactive score (IRS) was evaluate according to the methodology used in a previous article [7].

The same variables that were investigated in the previous article [7] together with IRS for D2-40, Gal-3, Maspin, and MCM7 were analyzed for each patient. The data was collected and stored in an Excel 2016 (Microsoft, USA) file. Due to the fast advancing of oral cancers, for survival, the patients were divided into two groups: group A - patients with survival less than one year, and group B patients with one year or more survival period. Similar, for the age variable, the patients were divided into two groups: group A, patients less or equal to 60 years, and group B, patients over 60 years old.

To obtain consistent statistics and data visualizations, the IRS were resampled, values over the score of 6 were considered Class B, while the remaining values were considered Class A. Having the classes resampled in Class A and B permitted a double statistic approach. While the initial scores were interpreted as numbers, the resulted classes were interpreted as categorical data. Seen as numerical data, the IRS values could be averaged, thus continuous statistics could be applied. Data normality was estimated using the Shapiro-Wilk test, and since all the data met the normal distribution criterion parametric tests were used: Student's $t$-test and analysis of variance (ANOVA) for two or more samples, respectively. The results were considered statistically significant if the $p$-value was $<0.05$.

In the previous paragraph, we described the use of numerical data for mean comparisons, but the numerical data also permitted correlation assessment. The correlations between the four IRSs were carried out using Pearson's $r$ correlation coefficient. The test value, $r$, is a variable ranging from -1 to 1 computed as the covariance of two variables normed by the product of their standard deviations (SDs). Positive values show direct correlation, negative values show inverse correlation, while values aiming at zero show no correlation. For a better understanding and for easy comparisons with other studies, $r$ 's intervals were empirically created as $[0 ; 0.2]$ - very weak, lack of correlation, $[0.2 ; 0.4]$ - weak correlation, $[0.4 ; 0.6]-$ reasonable correlation, $[0.6 ; 0.8]$ - high correlation, $[0.8 ; 1]$ - very high correlation, with the same interpretation on negative values.

Seen as categorical data, the IRS classes could be stratified and viewed as contingency tables, thus, the standard $\chi^{2}$ (chi-squared) statistics could be applied. Since the dataset was small and not all criteria of the $\chi^{2}$ test could be met, in these specific situations, the alternative Fisher's exact test was used. A $p$-value $<0.05$ was considered statistically significant for both tests.

The statistical assessment was carried out in Excel 2016 (Microsoft, USA) and in GraphPad Prism 9 (GraphPad, USA).

The present study was approved by the institutional Ethics Committee.

\section{ㅁ Results}

\section{D2-40 immunoexpression}

In the normal palatine epithelium adjacent to the tumors, the D2-40 was restricted to some of basal cells of squamous epithelia with both cytoplasmic and membranous pattern (Figure 1A). The reactivity was more intense and extended to the suprabasal cells in the dysplastic and hyperplastic epithelium adjacent to tumor proliferations (Figure 1B). In general, in the palatine squamous epithelium, at a distance from tumor proliferation, we did not show reactivity to D2-40. Also, intense reactivity with a predominantly membranous pattern was observed in the lymphatic vessels both from tumor and normal samples (Figure 1, A and C). Low reactivity was also noticed at the level of myoepithelial cells from minor salivary glands and nerve fibers that were present in the palatal mucosa.

The highest D2-40 tumor reactivity was observed in the poorly differentiated (G3) palate $\mathrm{SCC}$ cases (IRS $=9$ and IRS=12) with a diffuse expression and a dual subcellular pattern, membranous and cytoplasmic (Figure 1D). A slightly lower reactivity was recorded in the basaloid palate SCC cases (IRS=9) and moderately differentiated (G2) palate SCCs (with the median IRS=8). The expression pattern was similar to that from poorly differentiated cases, with a dominant membranous subcellular pattern that was more obvious at periphery of tumor proliferations and at the invasive front (Figure 1, E and F). Then, there were the papillary palate SCC cases (the IRS values were 6), closely followed by well-differentiated (G1) palate SCCs (with the median IRS=3). In these cases, the D2-40 reaction was restricted to the tumor cells from the periphery of neoplastic proliferation, with a dominant membranous subcellular pattern somewhat similar to reactivity from the palate epithelium adjacent to tumors (Figure 2, A and B). The lowest reactivity was recorded in the verrucous palate SCC (IRS=3) and in both cases of acantholytic SCC (IRS=2 and IRS=3). The pattern of reactions was similar to previous cases, being limited to tumor cells located at the interface with the stroma, the dominant subcellular pattern being the membranous one (Figure 2, C and D). In the acantholytic 
cases, the D2-40 reactivity was also present in some of the discohesive tumor cells with both cytoplasmic and membranous subcellular patterns (Figure 2E). Regardless of the HP subtype, the tumor reactivity to D2-40 was higher at the invasion front, immunostaining especially the tumor cells with the lowest degree of keratinization. A low reactivity was also recorded in the metastatic neoplastic proliferations from loco-regional lymph nodes (Figure 2F). This reactivity was more obvious in the least differentiated neoplastic cells from the periphery of tumor proliferations.
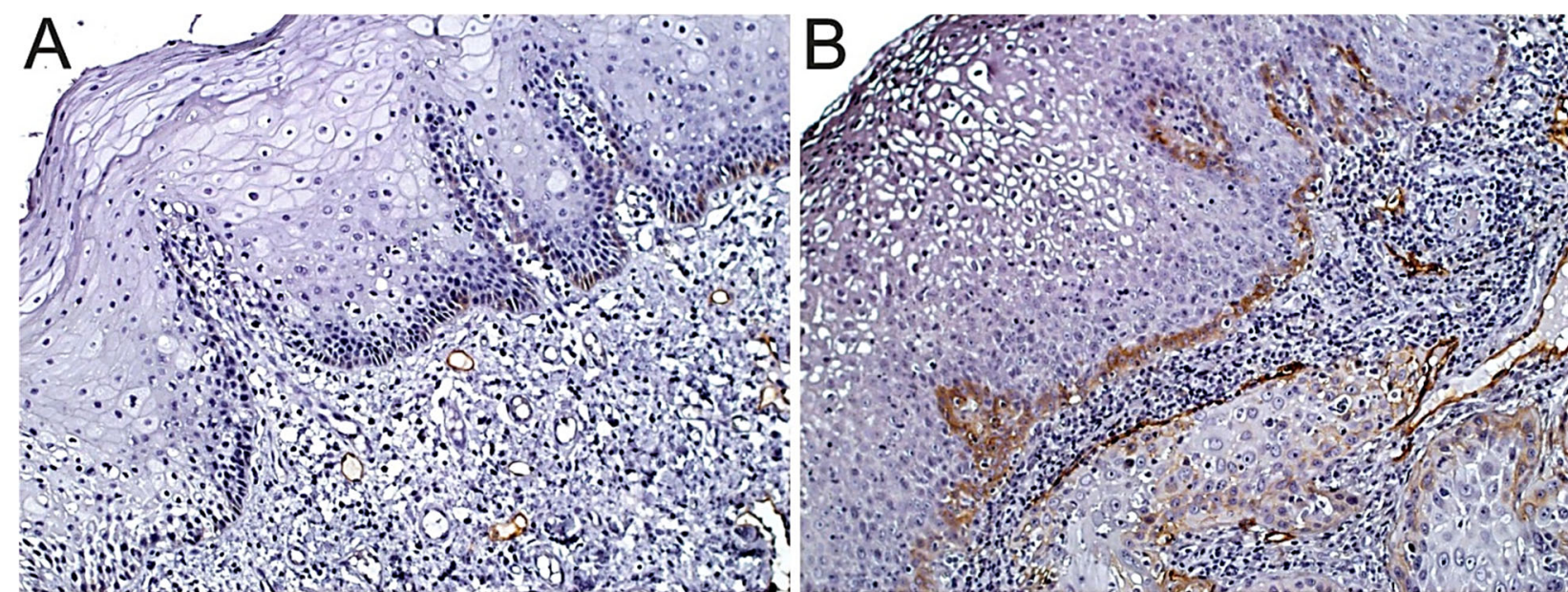

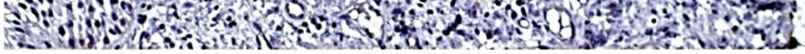
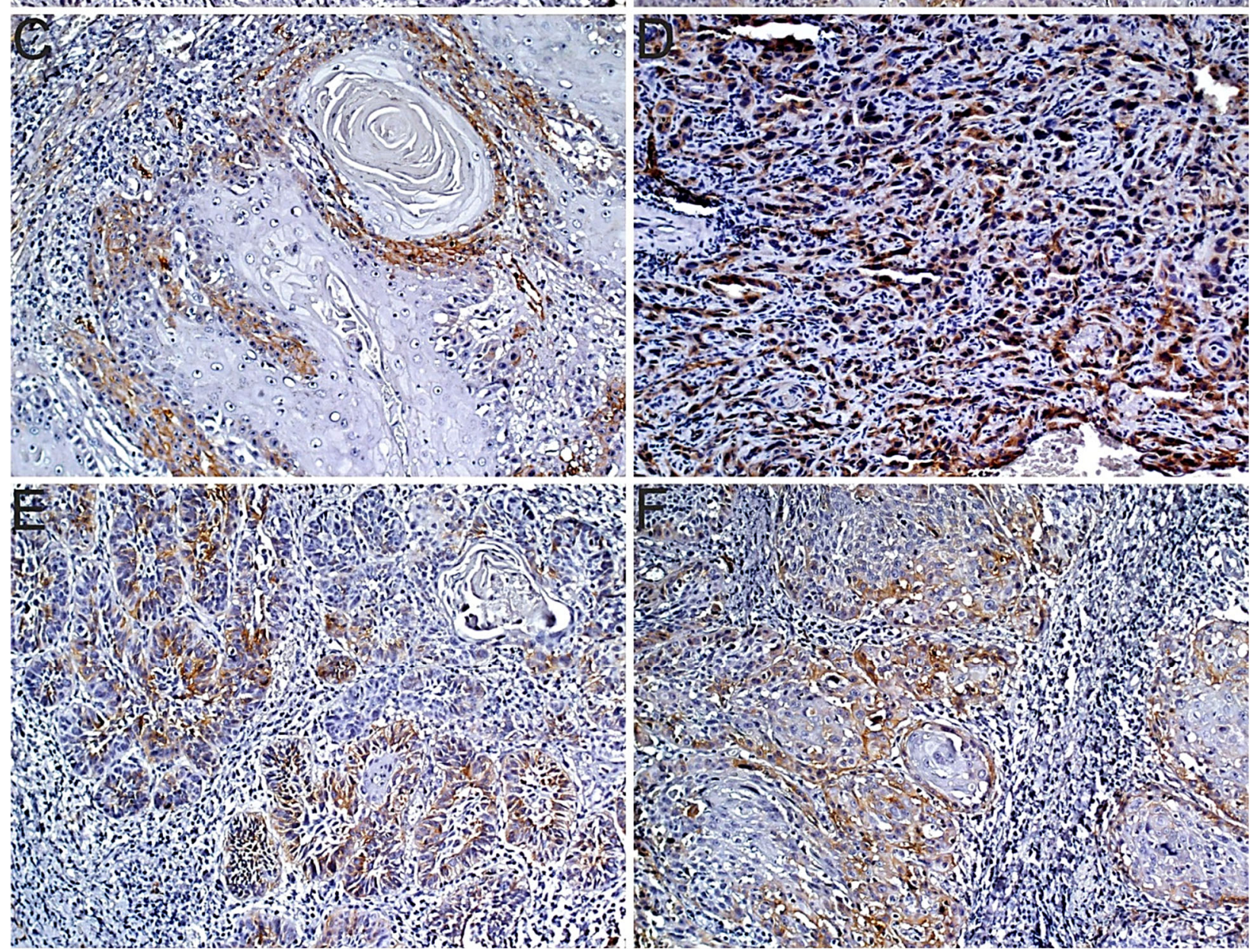

Figure 1 - D2-40 immunoreactivity: (A) At the level of basal layer from normal epithelium adjacent to the tumors with cytoplasmic and membrane pattern; $(B)$ The reactivity extended to the suprabasal layer in the hyperplastic and dysplastic epithelium; (C) Membranous reactivity in the lymphatic vessels both from tumor and normal samples; (D) The highest reactivity was noticed in the poorly differentiated (G3) palate SCC subtype; (E) This was followed by the basaloid variant, especially at the periphery of tumor proliferations; $(F) A$ similar reactivity was observed in moderately differentiated (G2) palate SCC subtype, more obvious at the invasive front. Anti-D2-40 antibody immunolabeling: $(A-F) \times 200$. SCC: Squamous cell carcinoma. 

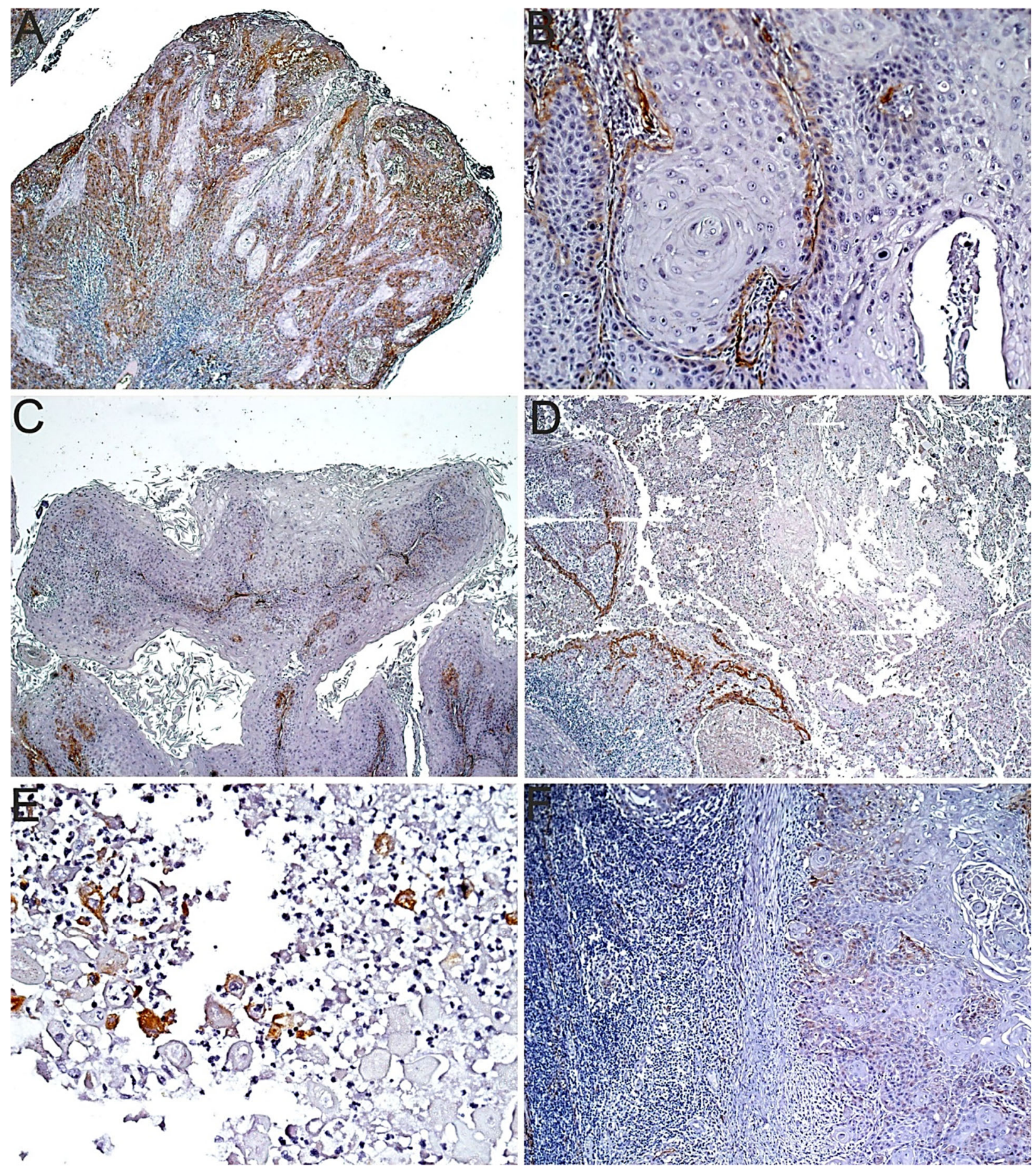

Figure 2 - D2-40 immunoreactivity: (A) An even lower reactivity was recorded in papillary palate SCC cases; (B) These were followed by the well-differentiated (G1) palate SCC cases where the reactivity was restricted to the tumor cells from the periphery of neoplastic proliferation, with a dominant membranous subcellular pattern; $(C)$ The lowest reactivity was recorded in the verrucous subtype; (D) This reactivity was similar to that of acantholytic SCC cases; (E) In the acantholytic cases, the D2-40 reactivity was also present in some of the discohesive tumor cells, with both cytoplasmic and membranous subcellular patterns; $(F) A$ lower reactivity was also recorded in the metastatic neoplastic proliferations from loco-regional lymph nodes. Anti-D2-40 antibody immunolabeling: $(A, C$ and $D) \times 50 ;(B) \times 200 ;(E) \times 400 ;(F) \times 100$. SCC: Squamous cell carcinoma.

\section{Gal-3 immunoexpression}

In the normal squamous mucosa of the palate, the Gal-3 reactivity was weak with nuclear pattern in basal and parabasal layers, and with cytoplasmic pattern in the superficial layers (Figure 3A). Intense reaction was noticed in the serous acini and ductal luminal cells of minor salivary glands with a mixed cytoplasmic, membranous, and nuclear pattern (Figure 3, B and C). Moderate to intense reactivity was also noticed in the fibroblasts, macrophages, smooth and skeletal muscle fibers, and nerve fibers with cytoplasmic and membranous pattern (Figure 3B).

In tumor tissue, the maximum reactivity was observed in the basaloid palate $\mathrm{SCC}$ cases (IRS=9 and IRS=12) with the nuclear pattern being the dominant one (Figure 3D). Then followed the cases of G3 palate SCCs (with a median IRS=9) 
which has a cytoplasmic dominant pattern, but with more intense reaction at the nuclear level (Figure 3E). A similar reactivity was present in the $\mathrm{G} 2$ tumors, but which overall had

a lower reactivity with a median value of IRS of 6 (Figure $3 F$ ).
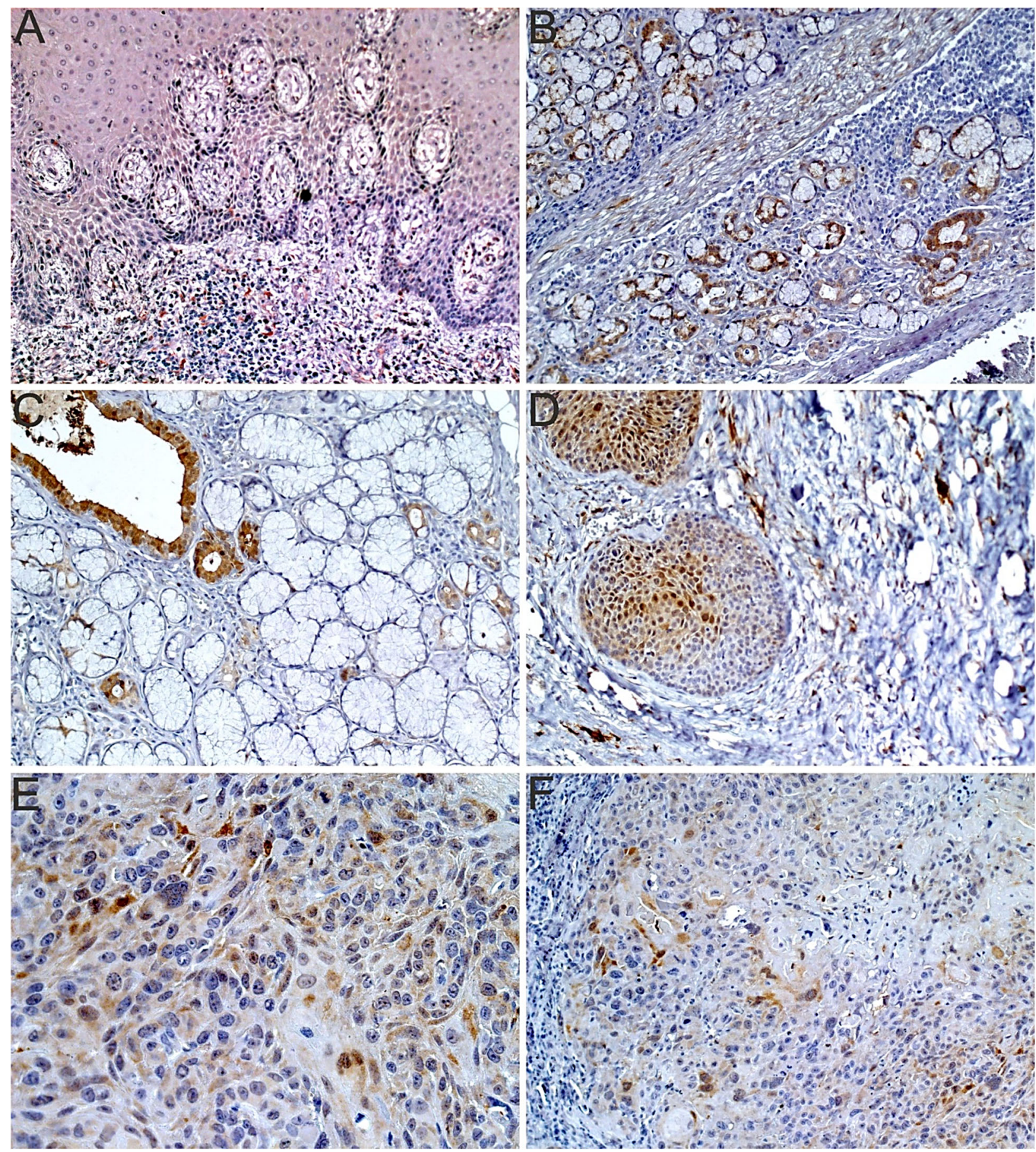

Figure 3 - Gal-3 immunoreactivity: (A) Weak nuclear reactivity in basal and parabasal layers, and with cytoplasmic pattern in the superficial layers of normal epithelium; (B) Intense reaction in the serous acini and ductal luminal cells of minor salivary glands with a mixed cytoplasmic, membranous, and nuclear pattern - also, a moderate reactivity was noticed in the nerve fibers with cytoplasmic and membranous pattern; (C) A strong cytoplasmic and membranous immunostaining at the level of the excretory units from minor salivary glands; (D) Maximum reactivity was observed in the basaloid palate SCC cases with prevalent nuclear pattern; (E) A slightly lower reactivity was observed in the G3 palate SCCs, which has a cytoplasmic dominant pattern, but with more intense reaction at the nuclear level; $(F) A$ similar reactivity was present in the G2 tumors. Anti-Gal-3 antibody immunolabeling: $(A-D$, and $F) \times 200 ;(E) \times 400$. G2: Moderately differentiated tumor; G3: Poorly differentiated tumor; Gal-3: Galectin-3; SCC: Squamous cell carcinoma.

In the acantholytic variant, the IRS values were 6 and 4, with the cytoplasmic pattern as the dominant one, this reactivity decreasing progressively towards the lumens (Figure 4A). At their level, we noticed that some of the tumor acantholytic cells retained the reactivity (Figure 4B).
A lower reactivity was recorded in G1 palate SCCs (the median IRS=2) with a prevalent cytoplasmic subcellular pattern, followed by the membranous one (Figure 4C). The lowest Gal-3 reactivity was observed in the verrucous palate SCC case $($ IRS $=2)$ and papillary palate $S C C s$ (IRS $=1$ and 
IRS $=2$ ) with a similar pattern as was noticed in G1 palate SCCs (Figure 4, D and E). Regardless of the HP subtype, the Gal-3 tumor reactivity was higher at the invasion front.

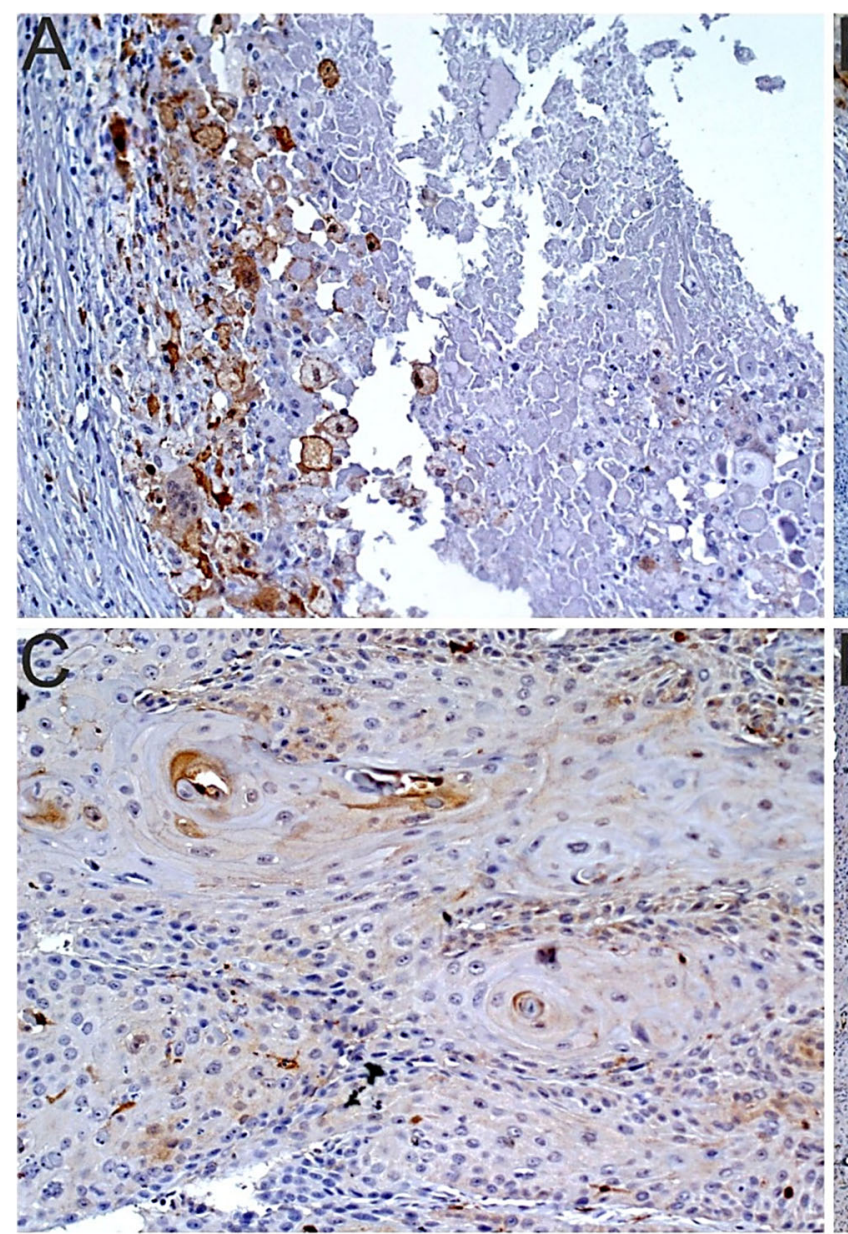

A low cytoplasmic reactivity was also noticed in metastatic tumor proliferations from the loco-regional lymph nodes (Figure 4F).
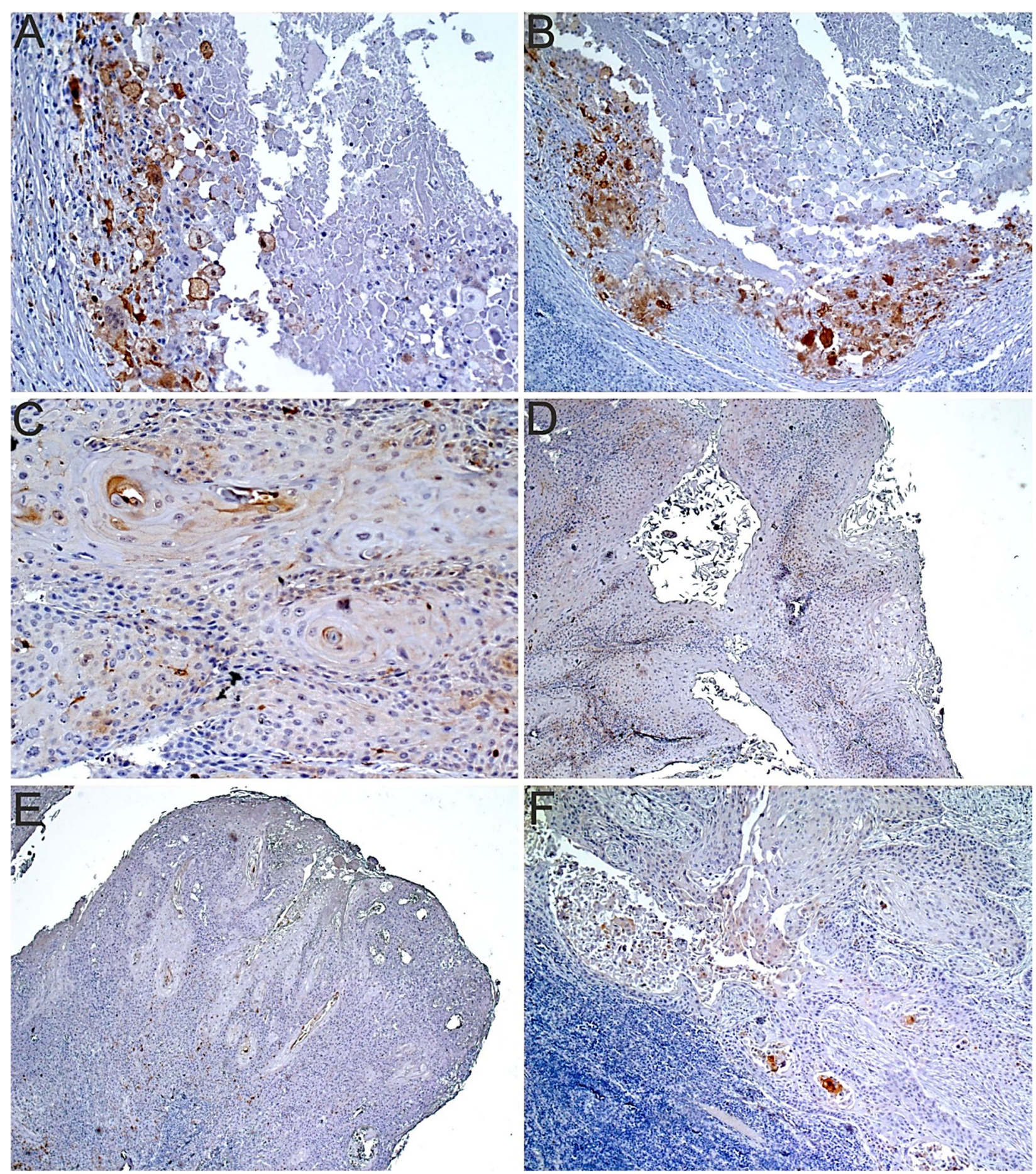

Figure 4 -Gal-3 immunoreactivity: (A) In the acantholytic variant, the cytoplasmic pattern was the dominant one, this reactivity decreasing progressively towards the lumens; (B) In the lumens, some tumor acantholytic cells retained the reactivity; (C) A lower reactivity was recorded in G1 palate SCCs, with a prevalent cytoplasmic subcellular pattern, followed by the membranous one; (D) The lowest Gal-3 reactivity was observed in the verrucous subtype; (E) This was followed by the papillary palate SCC that had a similar pattern as was noticed in G1 palate SCCs; (F) A lower cytoplasmic reactivity was also noticed in metastatic tumor proliferations from the loco-regional lymph nodes. Anti-Gal-3 antibody immunolabeling: $(A$ and $F) \times 100 ;(B$ and $C) \times 200 ;(D$ and $E) \times 50$. G1: Well-differentiated tumor; Gal-3: Galectin-3; SCC: Squamous cell carcinoma.

\section{Maspin immunoexpression}

The normal epithelium from the tumor samples showed a moderate cytoplasmic reactivity especially in the basal and suprabasal layers and also some diffuse nuclear reactivity in keratinocytes from the thickness of the epithelium (Figure 5A). A moderate cytoplasmic and nuclear reactivity at the level of the excretory units of the minor salivary 
glands with a high intensity in the myoepithelial cells. The skeletal fibers also presented a moderate cytoplasmic reactivity, and no reactivity was noticed neither in the chorion of the palatal mucosa nor in the tumor stroma.

The highest Maspin reactivity was observed in G1 cases (median IRS=9), with a diffuse cytoplasmic and membranous dominant pattern and focally also with a nuclear pattern (Figure 5B). The maximum reactivity was seen at the periphery of tumor proliferations diminishing towards keratin pearls. The palate SCC verrucous case showed a lower reactivity with the IRS value being 6 . The immunostaining pattern was similar to G1 palate SCCs (Figure 5C). Then followed the G2 palate SCCs (the median IRS=4) with similar immunostaining pattern as G1 palate SCCs (Figure 5D), but with an obvious decrease in reactivity to the invasion front (Figure 5E).
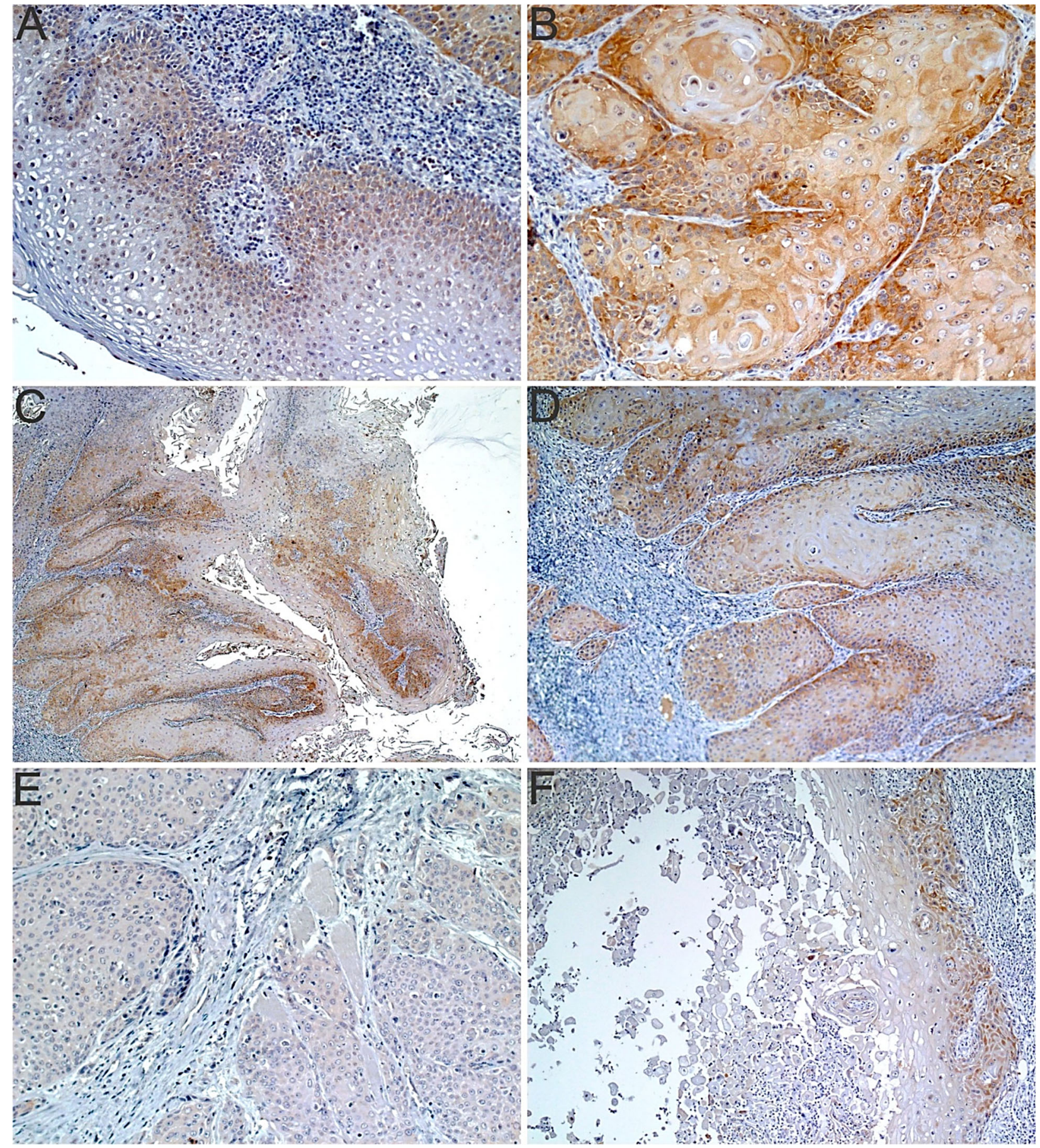

Figure 5 - Maspin immunoreactivity: (A) A moderate cytoplasmic reactivity especially in the basal and suprabasal layers and also some diffuse nuclear reactivity in keratinocytes from the thickness of the normal epithelium; (B) G1 palate SCCs were the most reactive, generally with a dominant cytoplasmic and membranous pattern but also focally with a nuclear pattern; (C) The verrucous case was less reactive; (D) These were followed by the G2 palate SCCs, with similar immunostaining pattern as was observed in G1 palate SCCs; (E) In these cases was obvious a decrease in reactivity to the invasion front; $(F) A$ similar reactivity was noticed in the acantholytic carcinomas, with an obvious decrease in reactivity in acantholysis areas. Anti-Maspin antibody immunolabeling: $(A, B$, and $E) \times 200 ;(C) \times 50 ;(D$ and $F) \times 100$. G1: Well-differentiated tumor; G2: Moderately differentiated tumor; Maspin: Mammary serine protease inhibitor; SCC: Squamous cell carcinoma. 
A similar reactivity was noticed in the acantholytic carcinomas (where the recorded IRS was 3 and 4) with an obvious decrease in reactivity in acantholysis areas (Figure 5F), where only a few acantholytic cells have retained Maspin reactivity (Figure 6A). In both papillary SCCs, the recorded IRS was 3 having a similar immunostaining pattern, as was observed in G2 palate SCCs (Figure 6B). Neoplastic cells from the papillae edges showed the highest reactivity (Figure 6C). In one of the two basaloid palate SCCs, no reactivity was evident, while in the other, an IRS value of 1 was recorded. In the latter case, a weak cytoplasmic reactivity was observed in neoplastic cells from the middle of tumor proliferations (Figure 6D). No Maspin reactivity was noticed in all G3 palate SCCs (Figure 6E). In those palate SCCs that developed lymph node metastases, a weak Maspin cytoplasmic reactivity was observed at the level of metastatic neoplastic proliferations (Figure 6F).
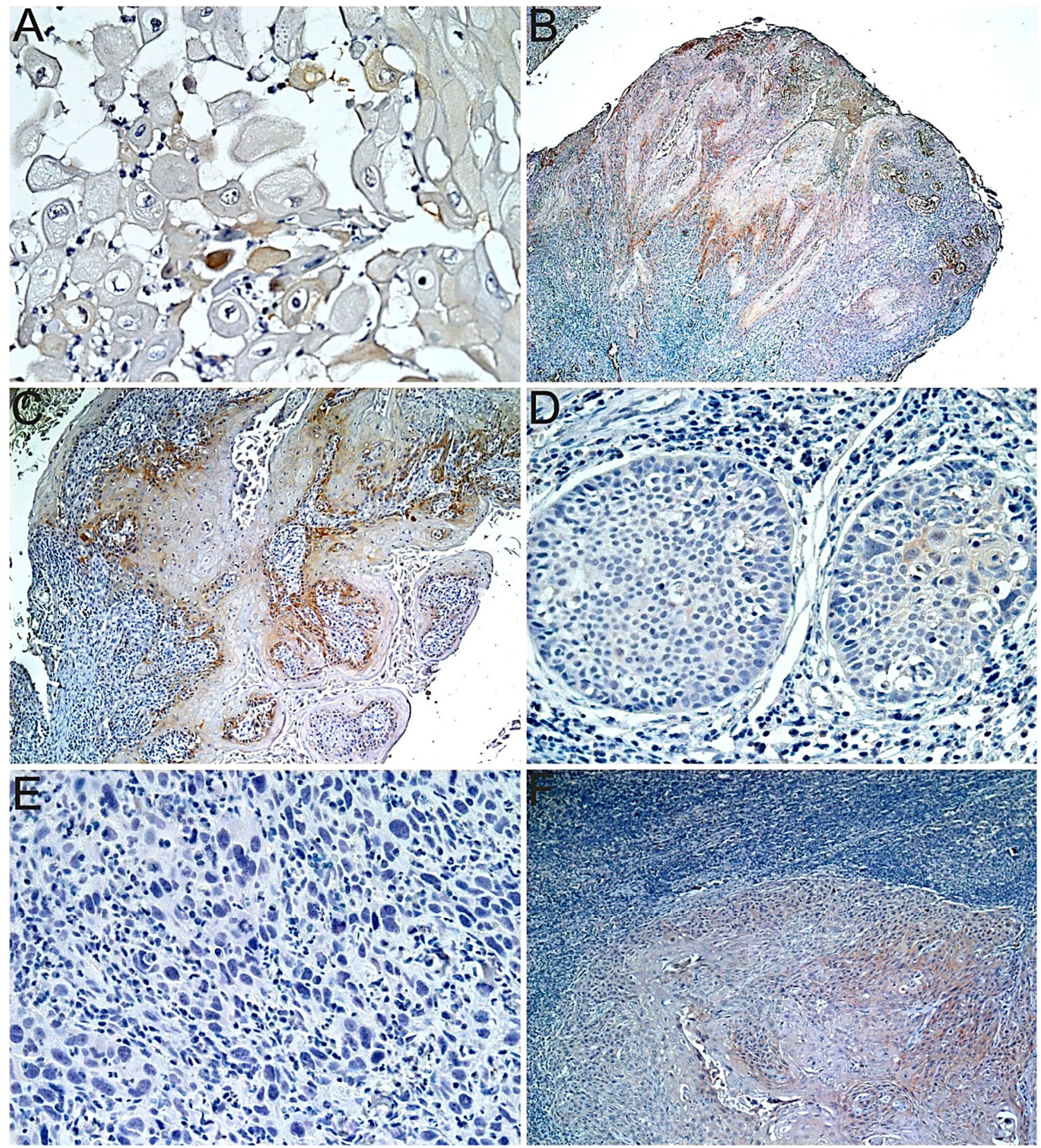

Figure 6 - Maspin immunoreactivity: (A) In the acantholysis areas, only a few acantholytic cells have retained Maspin reactivity; (B) The papillary SCCs had a similar immunostaining pattern as was observed in G2 palate SCCs; (C) The highest reactivity was observed in cells from the papillae periphery; (D) In one basaloid palate SCC, a weak cytoplasmic reactivity was observed in neoplastic cells from the middle of tumor proliferations; (E) No Maspin reactivity was noticed in all G3 palate SCCs; $(F) A$ weak Maspin cytoplasmic reactivity was observed at the level of metastatic neoplastic proliferations from lymph nodes metastases. Anti-Maspin antibody immunolabeling: $(A, D$, and E) $\times 400 ;(B) \times 50$; $(C$ and $F) \times 100$. G2: Moderately differentiated tumor; G3: Poorly differentiated tumor; Maspin: Mammary serine protease inhibitor; SCC: Squamous cell carcinoma. 


\section{MCM7 immunoexpression}

In the normal epithelium from tumor samples, the MCM7 reactivity was limited to the basal and parabasal layers heaving a nuclear pattern (Figure 7A). In the associated hyperplastic and dysplastic lesions, the reactivity increases towards the middle of the epithelium (Figure 7B). A lower nuclear reactivity was observed in some glandular cells from minor salivary gland and a high intensity in the germinal center cells from lymphoid follicles (Figure 7C).
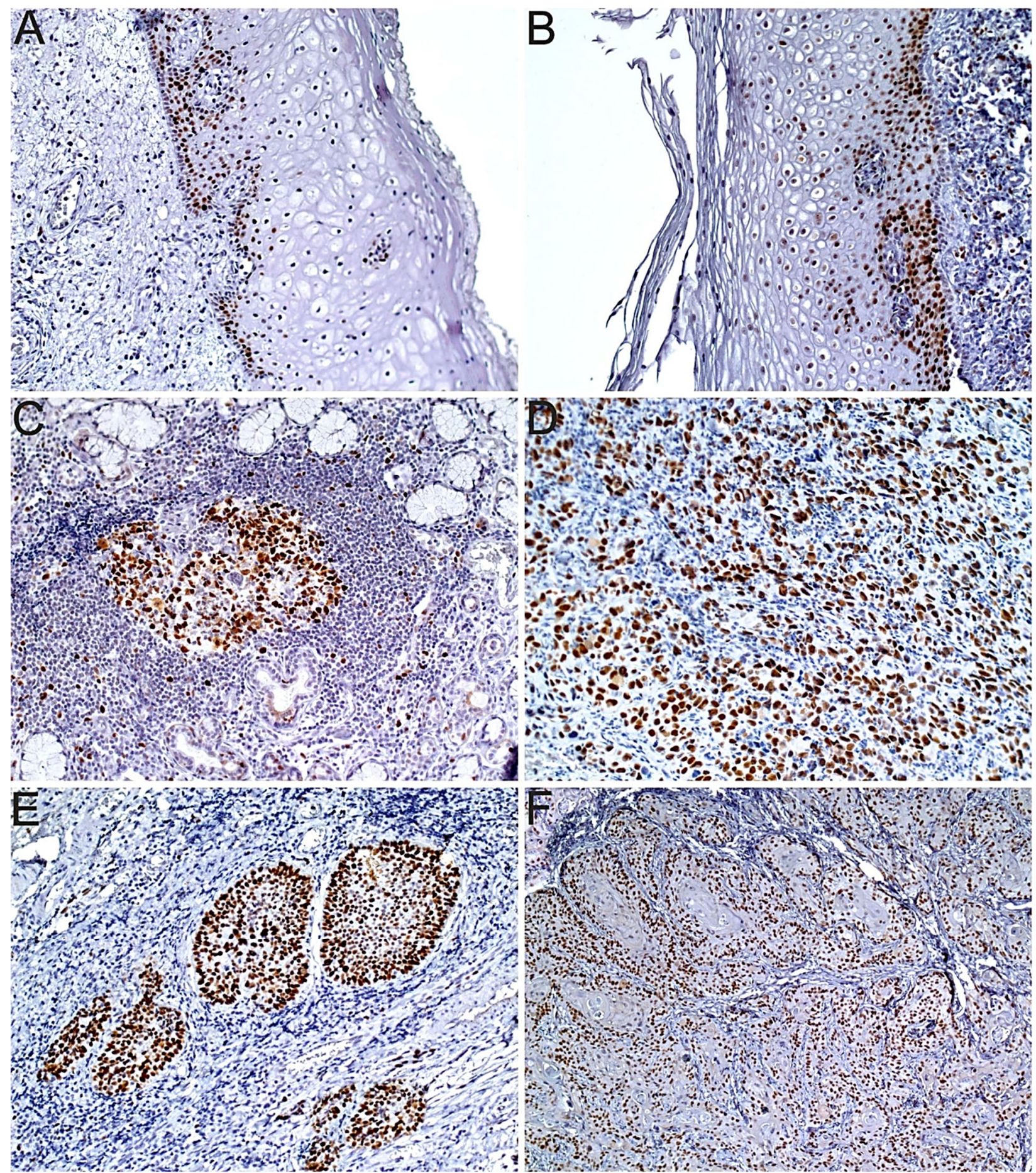

Figure 7-MCM7 immunoreactivity: (A) In the normal epithelium from tumor samples, the MCM7 reactivity was limited to the basal and parabasal layers heaving a nuclear pattern; (B) In the associated hyperplastic and dysplastic lesions, the reactivity increase towards the middle of the epithelium; (C) A lower nuclear reactivity was observed in some glandular cells from minor salivary gland and a high intensity in the germinal center cells from lymphoid follicles; (D) The highest $M C M 7$ reactivity was recorded in $G 3$ palate SCCs, with an exclusively nuclear pattern; (E) A similar immunostaining pattern was present in the basaloid subtype, the reactivity being less obvious towards the center of the tumor islands; (F) Then followed the G2 palate SCCs, with the highest reactivity in the cells from the periphery of tumor proliferations, the intensity of the immunostaining decreasing progressively inwards directly proportional to the degree of keratinization. Anti-MCM7 antibody immunolabeling: $(A-E) \times 200 ;(F) \times 100$. G2: Moderately differentiated tumor; G3: Poorly differentiated tumor; MCM7: Minichromosome maintenance complex component 7; SCC: Squamous cell carcinoma. 
In tumor tissue, the highest IRS values were noticed in G3 cases (median IRS=12), with an exclusively nuclear pattern (Figure 7D). A similar reactivity was noticed in the basaloid palate $\mathrm{SCCs}$ (IRS=8 and IRS=9), the marking being less obvious towards the center of the tumor islands (Figure 7E). Then followed the G2 palate SCCs, with a median IRS of 8 , with the highest reactivity in the cells from the periphery of tumor proliferations, the intensity of the immunostaining decreasing progressively inwards directly proportional to the degree of keratinization (Figure 7F).

A slightly lower reactivity was recorded in both papillary palate SCCs (IRS=4 and IRS=6) (Figure 8A). For the acantholytic palate SCCs, we recorded IRS values of 2 and 3 , the nuclear reactivity being present in the peripheral layers of acantholytic tumor islands (Figure 8B). Particularly, in this variant of palate SCC, there was a cytoplasmic reactivity in acantholytic cells (Figure $8 \mathrm{C}$ ). In the G1 palate SCCs, the median IRS value was of 2 , with the high intensity nuclear immunostaining in peripheral layers and no reactivity at the level of keratin pearls (Figure 8D). A similar reactivity was also noticed in the palate SCC verrucous case (IRS=2) (Figure 8E). A nuclear immunostaining reactivity was also recorded in the metastatic neoplastic proliferations from those palate SCCs that invaded loco-regional lymph nodes (Figure 8F).

\section{Statistical assessment}

Though some variables tended to stratify, the statistical inference did not show any differences for the following parameters: gender, age group, bone invasion, perineural invasion, and pain on all investigated immunomarkers.

All immunomarkers showed statistical stratification on localization (Figure 9), grading (Figure 10), muscle invasion, and survival. The D2-40, Gal-3, and MCM7 immunoexpression showed higher scores on the soft palate $v s$. hard palate, in opposition with the Maspin immunostaining. Similarly, grading stratification showed higher scores with increasing grade for D2-40, Gal-3, and MCM7, while for Maspin an opposite trend was shown as seen in Figure 10. The exact situation repeated in the muscle invasion and survival stratification where D2-40, Gal-3, and MCM7 were overexpressed in the tumors where muscles invasion was present and in patients with under one-year survival rate, while the Maspin tended to be underexpressed in these situations.

D2-40 showed statistical stratification with the tumor T score (Fisher's exact test statistical $p$-value was 0.0019 ; the result was significant at $p<0.05$ ). The continuous data analysis confirmed the localization, grading, and survival stratification from the categorical data analysis. In addition, stages 1 and 2 were statistically different (Student's $t$-test $p<0.05$ ) from stages 3 and 4 with mean (and SD) scores of $3.8 \pm 1.3,3.67 \pm 1,6.63 \pm 2.99$, and $8.53 \pm 3.02$, respectively.

With a similar behavior, Gal-3 showed statistical stratification with the tumor T score (Fisher's exact test statistical $p$-value was 0.00001 ; the result was significant at $p<0.05$ ), and node N score (Fisher's exact test statistical $p$-value was 0.0275 ; the result was significant at $p<0.05$ ), on the categorical data analysis. The findings were confirmed by the continuous data analysis. Staging had a better stratification, with all the four stages mean values being statistically different, ANOVA $p<0.001$, with individual Student's $t$-test between classes $p<0.003$. Reported mean (and SD) scores for stage were $1 \pm 0.01,2.22 \pm 0.44,4.63 \pm$ 1.96 , and $7.33 \pm 2.58$, respectively.

MCM7 behaved very similar with D2-40, showing stratification with tumor T score (Fisher's exact test statistical $p$-value was 0.0004; the result was significant at $p<0.05$ ), but also with node $\mathrm{N}$ score (Fisher's exact test statistical $p$-value was 0.0439; the result was significant at $p<0.05$ ). The similitude got even closer on the tumor $\mathrm{T}$ score, where the highest frequency of Class B tended to be present in the T3 and not in the T4 (Figure 11). The continuous data analysis confirmed the categorical data analysis and, similar with the D2-40, showed stage differences between stages 1,2 and 3, and 4.

Maspin's categorical data analysis showed statistical stratification with the tumor T score (Fisher's exact test statistical $p$-value was 0.0005 ; the result was significant at $p<0.05$ ), and with the node $\mathrm{N}$ score (Fisher's exact test statistical $p$-value was 0.0226 ; the result was significant at $p<0.05$ ), with better expression in lower tumor classes and in $\mathrm{N} 0$ and $\mathrm{N} 1$ node ones. Having a mirror effect with D2-40 and MCM7, the tumor T score where the highest frequency of Class B in class 2, and not in class 1 .

Correlation analysis confirmed that Maspin has an opposite behavior than the other three immunomarkers taken in consideration, with a reported $r$ of -0.8 with D2-40, - 0.9 with Gal-3, and -0.9 with MCM7, interpreted as very high inverse correlation. The other three immunomarkers have very high direct correlation, D2-40 r's with MCM7 having an incredible value of 1 , and 0.8 with Gal-3. In turn, Gal-3's correlation coefficient with MCM7 is 0.9 . These correlations sustain the similar findings from the localization, grading, muscle invasion, and survival stratification of scores.

\section{ㅁ Discussions}

Oral cancer is a global health problem as it is the $6^{\text {th }}$ to $8^{\text {th }}$ most common malignancy in the world $[8,9]$, and having a high mortality rate and leaving behind important morpho-functional disabilities [10]. $90 \%$ to $95 \%$ of oral cancers are oral SCC [11], which despite the latest progress in therapeutic management, still has poor prognosis, due to loco-regional aggressiveness and metastasis $[12,13]$. Local and regional recurrence are the key prognostic factor in oral SCC patients, to whom the overall five-year survival rates decreases from $92 \%$ in recurrence-free patients to $30 \%$ in patients with recurrence $[12,13]$. Data from the literature indicate that the survival rate of such patients seems to be influenced by the place where these tumors develop in the oral cavity, with oropharyngeal tumors and those that arise from the oral floor having the lowest survival rates [14]. Although rare, palate SCC appear to have increased local aggressively, easily invading adjacent muscle and bone structures and thereby leaving behind 
important morpho-functional defects [14]. In addition, the tumors developed at the level of the soft palate seem to be much more aggressive, the five-year overall survival rate dropping from $91 \%$ for the T1 tumors [15] to $33 \%$ in the case of T4 tumors [16]. Considering these particularities, in the present study, we investigated the IHC expression of the D2-40, Gal-3, Maspin and MCM7 immunomarkers, knowing that they play a major role in promoting locoregional cancer invasiveness.
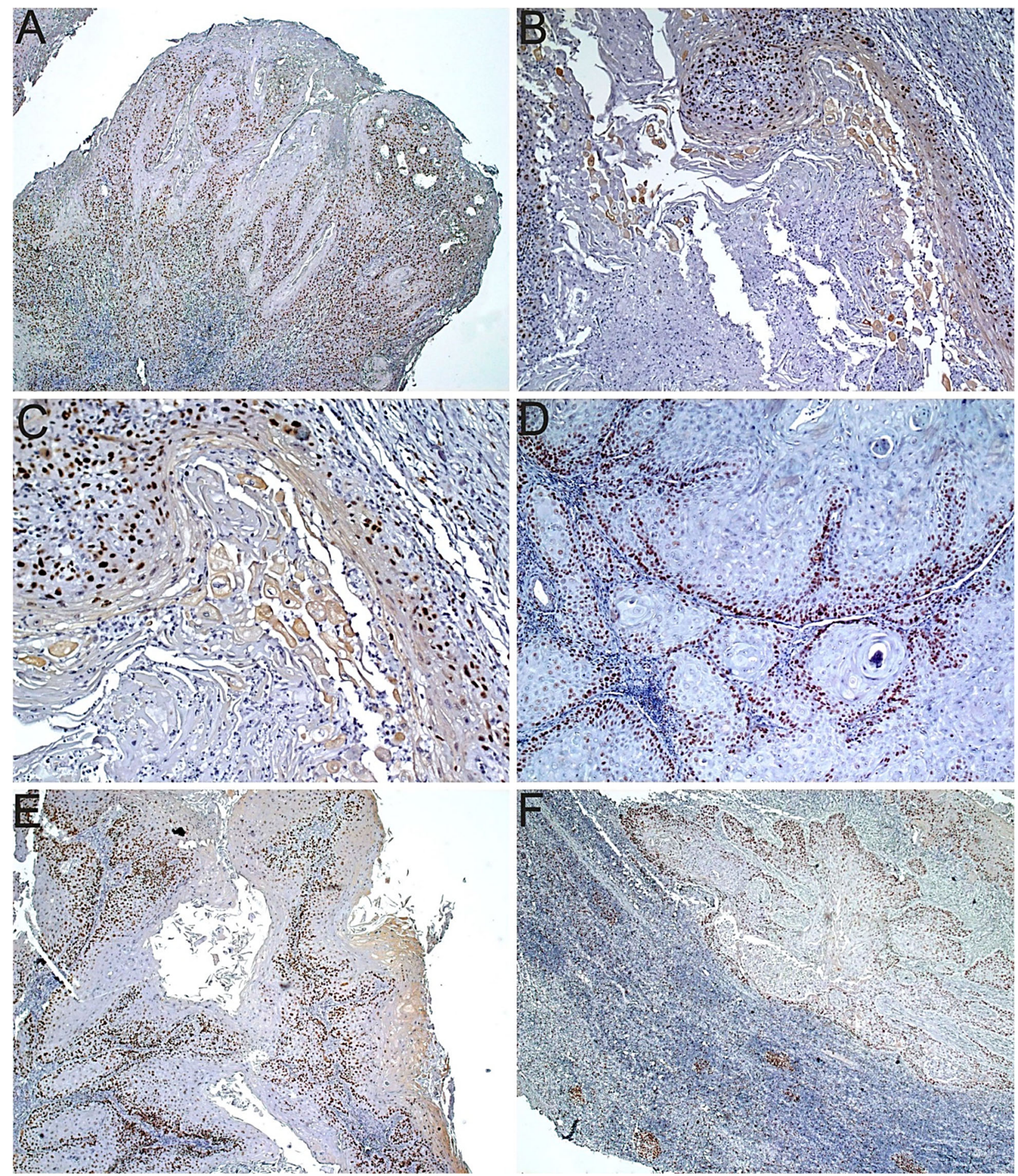

Figure 8-MCM7 immunoreactivity: (A) A slightly lower reactivity was recorded in both papillary palate SCCs; (B) In the acantholytic palate SCCs, the nuclear reactivity was present in the peripheral layers of acantholytic tumor islands; (C) Particularly, in this variant of palate SCC there was a cytoplasmic reactivity in acantholytic cells; (D) In the G1 palate SCCs, the high intensity nuclear immunostaining was noticed in peripheral layers and no reactivity at the level of keratin pearls; (E) A similar reactivity was also noticed in the palate SCC verrucous case; (F) A nuclear immunostaining reactivity was also recorded in the metastatic neoplastic proliferations from lymph nodes metastases. Anti-MCM7 antibody immunolabeling: $(A, E$ and $F) \times 50 ;(B$ and $D) \times 100 ;(C) \times 200$. G1: Well-differentiated tumor; MCM7: Minichromosome maintenance complex component 7; SCC: Squamous cell carcinoma. 


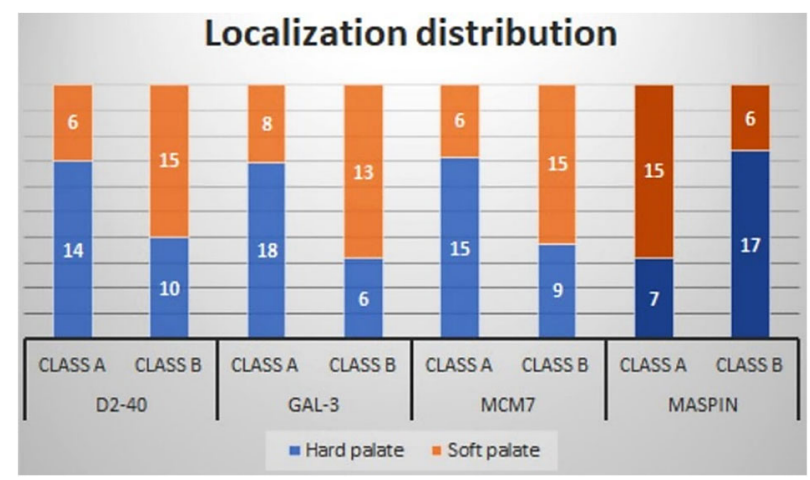

Figure 9-IRS values distribution on localization for all investigated markers. D2-40, Gal-3, MCM7 show a higher frequency of Class B (higher) score in the soft palate, while Maspin shows a higher frequency of Class A score. Class $A$ include the IRS values ranging from 1 to 6; Class $B$ comprise the IRS values higher than 6. D2-40: Podoplanin; Gal-3: Galectin-3; IRS: Immunoreactive score; Maspin: Mammary serine protease inhibitor; MCM7: Mini-chromosome maintenance complex component 7.

\section{Grading distribution}

Figure 10 - IRS values distribution on tumor grading for all investigated markers. D2-40, Gal-3, and MCM7 show predominant Class $A$ scores in $G 1$, combined Class $A$ and $B$ in $G 2$ and predominant Class $B$ in $G 3$, while Maspin scores are mirrored. Class $A$ include the IRS values ranging from 1 to 6; Class B comprise the IRS values higher than 6. D2-40: Podoplanin; G1: Well-differentiated tumor; G2: Moderately differentiated tumor; G3: Poorly differentiated tumor; Gal-3: Galectin-3; IRS: Immunoreactive score; Maspin: Mammary serine protease inhibitor; MCM7: Minichromosome maintenance complex component 7.

Tumor T score stratification

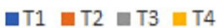

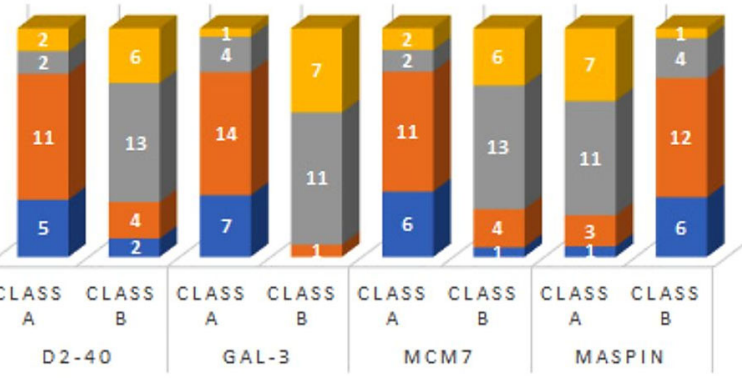

Podoplanin (D2-40)

Podoplanin (D2-40) is a 38-kDa type I transmembrane glycoprotein expressed in normal human tissues, such as kidney podocytes, lymphatic endothelium, skeletal muscle, alveolar type 1 cells, choroid plexus, glia cells, some type of neurons, fibroblasts, in the myofibroblasts of the breast and salivary glands, in the osteoblasts and mesothelial cells and in the basal layer of epidermis $[17,18]$. Under normal conditions, D2-40 has been shown to play an important role in lymphangiogenesis, platelet production in the bone marrow, and the immune response [19, 20], but its functions in other types of human tissues remain to be elucidated [18].

Podoplanin expression has been reported in a large variety of cancers, including angiosarcomas, malignant mesotheliomas, chondrosarcomas, osteosarcomas, gliomas, glioblastomas, germ-cell tumors, and SCCs with various locations, including in the oral cavity [20,21]. It seems that in these cancers Podoplanin promotes tumor progression especially by promoting the tumor cells migration, epithelialto-mesenchymal transition, remodeling of the extracellular matrix and cancer cell invasion $[22,23]$.

Our study revealed a gradual increase in D2-40 immunostaining during tumor progression from normal to dysplastic

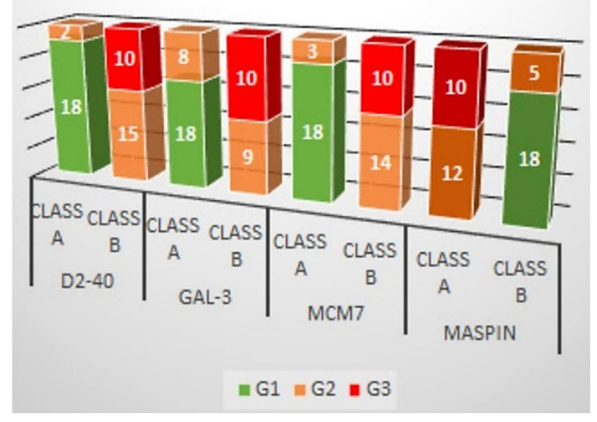

Figure 11 - IRS values distribution on T stage for all investigated markers. D2-40, Gal-3, and MCM7 show a similar distribution with a concentration of Class $A$ scores in $T 1$ and $T 2$, and concentration of Class $B$ scores in T3 and T4. Again, Maspin has a mirrored aspect with $T 1$ and $T 2$ concentration scores from Class B, and T3 and T4 from Class A. Class A include the IRS values ranging from 1 to 6; Class $B$ comprise the IRS values higher than 6. D2-40: Podoplanin;

Gal-3: Galectin-3; IRS: Immunoreactive score; Maspin: Mammary serine protease inhibitor; MCM7: Minichromosome maintenance complex component 7.

epithelium, respective to palate SCCs. From a dominant membranous and cytoplasmic pattern seen in the basal and parabasal cells, the D2-40 reactivity has expanded to superficial layers in hyperplastic and dysplastic lesions were the cytoplasmic pattern was dominant. In tumor samples, the highest reactivity was noticed in poorly differentiated (G3) palate SCC cases, with the cytoplasmic pattern as most prevalent. The lowest reactivity was recorded in the verrucous and well-differentiated (G1) palate SCCs, with a dominant membranous subcellular pattern that was more obvious at periphery of tumor proliferations. Regardless of the HP subtype, the tumor reactivity to D2-40 was higher at the invasion front, immunostaining especially the tumor cells with the lowest degree of keratinization. A weak D2-40 immunoexpression was also noticed in the metastatic neoplastic proliferations from loco-regional lymph nodes, with the reactivity being more evident especially at the level of less differentiated neoplastic proliferations.

The first evidence of Podoplanin expression in basal cells from hyperplastic and dysplastic epithelial areas associated to oral cancer was provided by Yuan et al. (2006) [24]. Then followed a series of other articles that showed Podoplanin expression in dysplastic cells from premalignant oral lesions $[25,26]$. It has been speculated 
that the extension of Podoplanin expression in the suprabasal layers would in fact represent the clonal expansion of stem cells during oral carcinogenesis, suggesting the certain potential for malignant transformation of such lesions [27]. Several authors have shown that Podoplanin expression increased with the severity of dysplasia, recognizing its role as a predictive factor in the malignant progression of such lesions [25-28].

Most of the studies indicate a fairly high percentage of oral SCCs (from $84 \%$ to $100 \%$ ) expressing Podoplanin $[17,25,29]$, some of them suggesting that the level of expression is inversely correlated with the degree of differentiation of these tumors [25, 28, 29]. At the same time, it was shown that Podoplanin expression was increased in the neoplastic cells from the periphery of tumor proliferations suggesting that these cells would have a greater capacity for proliferation and renewal [17, 29, 30]. Only one study found a higher expression of Podoplanin in the tongue and oral floor SCCs compared with other oral tumor sites [31]. Moreover, the authors suggested that this expression would justify a higher rate of incidence of loco-regional lymph node metastases for such localizations of oral SCCs. Most of authors showed a strong association between Podoplanin expression and lymph node metastasis rate [31-33].

Regarding the prognostic role of Podoplanin expression, opinions are divided, some denying this role [31, 34], while others have shown that a high level of expression would be associated with a poorer prognosis [24, 32, 33] making from this marker a potential therapeutic target [35].

\section{Gal-3}

Gal-3 belongs to the $\beta$-galactoside-binding lectins family, being involved in cell growth, apoptosis, cell adhesion, differentiation, transformation, angiogenesis, inflammation, fibrosis, host defense, tumorigenesis, and metastasis [36, 37]. This marker has been shown to be overexpressed in tumor cells and tumor-related stromal cells, which is why it can be an effective molecular target in treating cancer [38-40].

Our investigation recorded a positive Gal-3 reaction in the normal oral mucosa adjacent to tumor proliferations with a nuclear dominant pattern in basal and parabasal layers, and with a cytoplasmic pattern which was most prevalent in the superficial layers. In tumor tissue, the maximum immunoreactivity was recorded in the basaloid and G3 cases, with nuclear pattern as the dominant one, while for the verrucous and G1 palate SCCs, we recorded the lowest Gal-3 immunoreactivity with a cytoplasmic and membranous patterns as mist prevalent. Regardless of the HP subtype, the Gal-3 tumor reactivity was higher at the invasion front and a low reactivity was also noticed in metastatic tumor proliferations from the loco-regional lymph nodes. Also, some stromal reactivity was also noticed in the fibroblasts and macrophages from tumor samples.

Some authors have shown that during neoplastic progression, at the level of tongue mucosa, the Gal-3 protein is translocated from cytoplasm to nucleus, and this may serve as a prognostic factor for tongue SCC patients [41]. Many authors found a direct correlation between Gal-3 expression and tumor grade and lymph node involvement for oral and head and neck SCCs [4245]. Regarding the tumor stage, opinions are divided, while some showed the existence of a correlation with the size of the tumor [44, 45], others did not find such a correlation [42, 43]. Plzák et al. (2004) recorded in head and neck SCCs a significant correlation between Gal-3 reactivity and $\mathrm{HP}$ grade, tumor keratinization, and $\mathrm{N}$ stage and no relationship with age, primary tumor topography, and $T$ stage or clinical stage [42]. Weber et al. (2017) conferred to Gal-3 a possible tumorigenic role in the oral squamous epithelium [45]. On the other hand, Wang et al. (2013) suggested that Gal-3 play a key role in the progression of tongue SCCs through activation of the $\mathrm{Wnt} / \beta$-catenin signaling pathway [44]. Also, while some authors have failed to find correlation between Gal-3 expression and disease progression in oral SCCs $[43,46]$, other established that enhanced expression of cytoplasmic Gal-3 is an independent predictor of disease recurrence [41]. Moreover, Wehrhan et al. (2018) recorded an increased Gal-3 expression in regional lymph nodes of large oral SCCs suggesting that blocking of this marker in the early-stage of such tumors might be a therapeutic option in oral cancer [47].

\section{Maspin}

Maspin is a member of the serine protease inhibitor/ non-inhibitor superfamily (serpin) being expressed in epithelia especially in the nucleus of normal cells $[48,49]$. It has been shown that downregulation or translocation of Maspin from the nucleus to the cytoplasm correlated with a poor prognosis and a low survival of cancer patients [50-52]. These being explained by the fact that Maspin is a proteases inhibitor which prevents metastasis and tumor progression controlling apoptosis of tumor cells and also the tumor angiogenesis [53, 54].

Investigated the Maspin reactivity, we found a moderate cytoplasmic reactivity especially in the basal and suprabasal layers of the normal epithelium and also some diffuse nuclear reactivity in keratinocytes from the thickness of the epithelium. The highest Maspin immunoreactivity was noticed in G1 cases with a diffuse cytoplasmic and membranous dominant pattern and focally also with a nuclear pattern. No Maspin reactivity was noticed in all investigated G3 palate SCCs. Regardless HP variant, we also observed a decrease of Maspin reactivity to the invasion front and a weak reactivity was noticed in the lymph node metastatic proliferations.

In some studies, regarding the expression of Maspin in oral SCC, it has been shown that its expression correlated with a better survival rate but instead no correlation was found with differentiation degree, type of invasion, Tstage, and lymph-node metastasis [55-57]. At the same time, it was shown that cytoplasmic expression was associated with a high risk of neck lymph node metastasis in oral SCCs [58]. Similarly, other authors have shown that Maspin decreased expression in oral SCCs was associated with an unfavorable clinical outcome due to invasion and lymph node metastasis $[59,60]$. Somewhat in contradiction, it was reported that Maspin expression did not correlate with survival in oral tongue SCCs, suggesting that as the tumor grows and becomes more invasive it would occur a downregulation of Maspin expression [61]. The authors 
also suggested that the $p 53$ gene could be a Maspin target and thereby contribute to the inhibition of tumor angiogenesis. In addition, it has been shown that Maspin expression can be a predictor of the response to chemoradiotherapy in patients with head and neck SCCs [58]. Thus, it was shown that higher Maspin expression was significant statistically correlated with longer disease-free survival and longer overall survival and disease-specific survival in such cancer patients after chemoradiotherapy.

\section{MCM7}

MCM7 is a member of a family of six structurally related proteins, called minichromosome maintenance protein complex (MCM) that possess helicase activity [62]. These proteins play important roles in initiation and elongation during deoxyribonucleic acid (DNA) replication being expressed only in replicating cells $[62,63]$. Overexpression of MCMs was recorded in various cancers [64-66] being a promising marker of cell proliferation with higher sensitivity than traditional cell-cycle markers, being present in all stages of the cell division cycle and which seem to be much less influenced by external factors like inflammatory factors $[64,66]$.

Our study revealed an increase in the MCM7 reactivity from the normal epithelium where the reaction was limited to the basal and parabasal layers heaving a nuclear pattern to the hyperplastic and dysplastic lesions were the immunostaining extended towards the middle of the epithelium. In tumor tissue the highest MCM7 immunoreactivity was recorded in $\mathrm{G} 3$ cases with an exclusively diffuse nuclear pattern. On the contrary, in G1 cases was noticed the lowest reactivity with the high intensity nuclear immunostaining in peripheral layers and no reactivity at the level of keratin pearls. Also, we noticed a higher reactivity at the periphery of tumor proliferations and also at the invasion front. The nuclear immunostaining reactivity was also observed in the metastatic neoplastic proliferations from those palate SCCs that invaded loco-regional lymph nodes.

Reviewing data from the literature, we found few studies on MCM7 expression in oral cancer. Thus, Feng et al. (2008) recorded MCM7 expression in the normal oral mucosa epithelium restricted to the basal layer and 1-2 layers above it, indicating the presence of cell division only in these layers [67]. Also, the authors proved a progressive MCM7 overexpression from normal epithelium to moderate-severe dysplasia and invasive carcinoma, suggesting an increase in the number of cells that divide during oral carcinogenesis. The authors concluded that the level of MCM7 protein expression is a direct indicator of the cellular proliferation and can be considered a diagnostic marker of oral SCCs $v s$. oral precancerous lesions. Also, the level of MCM7 expression from oral cancers correlated with lymph node metastasis and clinical TNM stage, so this marker may also have a prognostic role, its overexpression indicating cases with a poor prognosis [67]. A direct correlation was also observed between the expression of MCM7 and cell division cycle 6 (Cdc6), suggesting that the two proteins could be useful molecular markers in the early diagnosis and prediction of the prognosis among patients with oral SCCs. Tamura et al. (2010) also highlighted a progressive increase in the level of MCM7 expression from normal epithelium to dysplastic lesions and finally to oral SCCs [68]. The authors recorded a high MCM7 expression in poorly differentiated oral SCC compared to well- or moderately differentiated oral SCC and overall, its expression in oral SCC specimens was higher than that from normal and dysplastic oral mucosa. In addition, was recorded a statistically significant correlation between MCM7 expression and geminin and Ki-67 expression in oral SCCs [68]. Therefore, this study proved that MCM7 expression was correlated with HP grade and unfavorable prognosis of oral SCCs, suggesting that this marker together with geminin would be reliable prognostic markers for such cancer patients.

Statistically, we found significant stratification on localization, grading, muscle invasion, and survival for all investigated markers. The highest IRS values were recorded for D2-40, Gal-3, and MCM7 in opposition to Maspin, for those tumors developed from the soft palate, that were poorly differentiated, which had. Moreover, the D2-40 showed statistical stratification with the T stage, while Gal-3 and MCM7 and showed the same tendency regarding the $\mathrm{T}$ stage, but also for the $\mathrm{N}$ stage. For Maspin was noticed a similar but opposite tendency as was observed for Gal-3 and MCM7 immunostaining. Overall, between D2-40, Gal-3, and MCM7, IRS values were very high direct correlations, while between Maspin and each of the previous markers there were very high inverse correlations.

\section{a Conclusions}

We found a progressive increase in reactivity for D2-40 and MCM7 from the normal epithelium toward dysplastic epithelium and respectively to SCC, which suggests the intervention of these immunomarkers in the early stages of squamous cell carcinogenesis in the palate. The highest D2-40, Gal-3 and MCM7 immunoreactivity was noticed in basaloid and G3 palate SCCs, while for the Maspin, the G1 palate SCCs were the most reactive. The first three immunomarkers mentioned above were most intensely expressed at the invasion front, while the Maspin immunoreactivity was low or absent at this level. Statistically, we found significant stratification on localization, grading, muscle invasion, and survival for all investigated immunomarkers, but with very high direct correlations between D2-40, Gal-3, and MCM7 IRS values, while between the Maspin and each of the previous immunomarkers there were very high inverse correlations. All these investigated immunomarkers proved to be responsible for the local invasiveness and regional lymph node metastasis, thus allowing a prognostic and therapeutic stratification of patients with palate SCCs.

\section{Conflict of interests}

The authors declare that there is no conflict of interests regarding the publication of this paper. All authors read and approved the final manuscript.

\section{Acknowledgments}

We are grateful to Mircea-Sebastian Şerbănescu, Lecturer, MD, PhD, Department of Medical Informatics and Biostatistics, University of Medicine and Pharmacy of Craiova, Romania for the support with statistical analysis and interpretation. 


\section{References}

[1] Bray F, Ferlay J, Soerjomataram I, Siegel RL, Torre LA, Jemal A Global cancer statistics 2018: GLOBOCAN estimates of incidence and mortality worldwide for 36 cancers in 185 countries. CA Cancer J Clin, 2018, 68(6):394-424. https://doi.org/10.3322/ caac.21492 PMID: 30207593

[2] Kim JW, Park Y, Roh JL, Cho KJ, Choi SH, Nam SY, Kim SY. Prognostic value of glucosylceramide synthase and P-glycoprotein expression in oral cavity cancer. Int J Clin Oncol, 2016 21(5):883-889. https://doi.org/10.1007/s10147-016-0973-1 PMID: 27000845

[3] Farhood Z, Simpson M, Ward GM, Walker RJ, OsazuwaPeters N. Does anatomic subsite influence oral cavity cancer mortality? A SEER database analysis. Laryngoscope, 2019 129(6):1400-1406. https://doi.org/10.1002/lary.27490 PMID: 30408182

[4] Brierley JD, Gospodarowicz MK, Wittekind C (eds). TNM classification of malignant tumors. $8^{\text {th }}$ edition, Union for International Cancer Control (UICC), Willey-Blackwell, John Wiley \& Sons, Ltd., 2017, 272. https://www.wiley.com/en-vn/ TNM+Classification+of+Malignant+Tumours $\% 2 \mathrm{C}+8$ th+Editionp-9781119263579

[5] Urken ML. Advances in head and neck reconstruction. Laryngoscope, 2003, 113(9):1473-1476. https://doi.org/10. 1097/00005537-200309000-00008 PMID: 12972917

[6] Pătru A, Şurlin V, Mărgăritescu C, Ciucă E, Mărgăritescu OC, Camen A. Palate squamous cell carcinomas: a ten-year single institute experience. Curr Health Sci J, 2020, 46(4):358-370. https://doi.org/10.12865/CHSJ.46.04.06 PMID: 33717510 PMCID: PMC7948021

[7] Pătru A, Şurlin V, Mărgăritescu C, Ciucă EM, Matei M Şerbănescu MS, Camen A. Analysis of the distribution and expression of some tumor invasiveness markers in palate squamous cell carcinomas. Rom J Morphol Embryol, 2020 , 61(4):1259-1278. https://doi.org/10.47162/RJME.61.4.27 PMID: 34171074

[8] D'Cruz AK, Vaish R, Dhar H. Oral cancers: current status. Oral Oncol, 2018, 87:64-69. https://doi.org/10.1016/j.oralon cology.2018.10.013 PMID: 30527245

[9] Ferlay J, Soerjomataram I, Dikshit R, Eser S, Mathers C Rebelo M, Parkin DM, Forman D, Bray F. Cancer incidence and mortality worldwide: sources, methods and major patterns in GLOBOCAN 2012. Int J Cancer, 2015, 136(5):E359-E386. https://doi.org/10.1002/ijc.29210 PMID: 25220842

[10] Omura K. Current status of oral cancer treatment strategies: surgical treatments for oral squamous cell carcinoma. Int J Clin Oncol, 2014, 19(3):423-430. https://doi.org/10.1007/s1 0147-014-0689-z PMID: 24682763

[11] Al-Jaber A, Al-Nasser L, El-Metwally A. Epidemiology of ora cancer in Arab countries. Saudi Med J, 2016, 37(3):249-255. https://doi.org/10.15537/smj.2016.3.11388 PMID: 26905345 PMCID: PMC4800887

[12] Liu CH, Chen HJ, Wang PC, Chen HS, Chang YL. Patterns of recurrence and second primary tumors in oral squamous cell carcinoma treated with surgery alone. Kaohsiung J Med Sci, 2013, 29(10):554-559. https://doi.org/10.1016/j.kjms.2013.03. 001 PMID: 24099110

[13] Wang B, Zhang S, Yue K, Wang XD. The recurrence and survival of oral squamous cell carcinoma: a report of 275 cases. Chin J Cancer, 2013, 32(11):614-618. https://doi.org/10.5732/ cjc.012.10219 PMID: 23601241 PMCID: PMC3845544

[14] American Cancer Society (ACS). Survival rates for oral cavity and oropharyngeal cancer. ACS, Inc.; last revised: February 2, 2021; accessed: March 23, 2021. https://www.cancer.org/ cancer/oral-cavity-and-oropharyngeal-cancer/detection-diag nosis-staging/survival-rates.html https://www.cancer.org/con tent/dam/CRC/PDF/Public/8765.00.pdf

[15] Douglas WG, Rigual NR, Giese W, Bauer J, Wiseman SM, Loree TR, Schwarz J, Alrawi S, Hicks WL Jr. Advanced soft palate cancer: the clinical importance of the parapharyngeal space. Otolaryngol Head Neck Surg, 2005, 133(1):66-69. https:// doi.org/10.1016/j.otohns.2005.03.007 PMID: 16025055

[16] Hansen E, Panwala K, Holland J. Post-operative radiation therapy for advanced-stage oropharyngeal cancer. J Laryngol Otol, 2002, 116(11):920-924. https://doi.org/10.1258/00222 150260369462 PMID: 12487672
[17] Prasad B, Kashyap B, Babu GS, Kumar GR, Manyam R. Expression of podoplanin in different grades of oral squamous cell carcinoma. Ann Med Health Sci Res, 2015, 5(4):299-304. https://doi.org/10.4103/2141-9248.160181 PMID: 26229720 PMCID: PMC4512124

[18] Quintanilla M, Montero-Montero L, Renart J, Martín-Villar E. Podoplanin in inflammation and cancer. Int J Mol Sci, 2019, 20(3):707. https://doi.org/10.3390/ijms20030707 PMID: 30736372 PMCID: PMC6386838

[19] Astarita JL, Acton SE, Turley SJ. Podoplanin: emerging functions in development, the immune system, and cancer. Front Immunol, 2012, 3:283. https://doi.org/10.3389/fimmu.2012.00283 PMID: 22988448 PMCID: PMC3439854

[20] Renart J, Carrasco-Ramírez P, Fernández-Muñoz B, MartínVillar E, Montero L, Yurrita MM, Quintanilla M. New insights into the role of podoplanin in epithelial-mesenchymal transition. Int Rev Cell Mol Biol, 2015, 317:185-239. https://doi.org/10. 1016/bs.ircmb.2015.01.009 PMID: 26008786

[21] Ordóñez NG. Value of podoplanin as an immunohistochemical marker in tumor diagnosis: a review and update. Appl Immunohistochem Mol Morphol, 2014, 22(5):331-347. https://doi.org/ 10.1097/PAI.0b013e31828a83c5 PMID: 23531849

[22] Wicki A, Lehembre F, Wick N, Hantusch B, Kerjaschki D, Christofori G. Tumor invasion in the absence of epithelialmesenchymal transition: podoplanin-mediated remodeling of the actin cytoskeleton. Cancer Cell, 2006, 9(4):261-272. https:// doi.org/10.1016/j.ccr.2006.03.010 PMID: 16616332

[23] Li YY, Zhou CX, Gao Y. Podoplanin promotes the invasion of oral squamous cell carcinoma in coordination with MT1MMP and Rho GTPases. Am J Cancer Res, 2015, 5(2):514529. PMID: 25973294 PMCID: PMC4396046

[24] Yuan P, Temam S, El-Naggar A, Zhou X, Liu DD, Lee JJ, Mao L. Overexpression of podoplanin in oral cancer and its association with poor clinical outcome. Cancer, 2006, 107(3): 563-569. https://doi.org/10.1002/cncr.22061 PMID: 16804930

[25] Inoue H, Miyazaki Y, Kikuchi K, Yoshida N, Ide F, Ohmori Y, Tomomura A, Sakashita H, Kusama K. Podoplanin expression during dysplasia-carcinoma sequence in the oral cavity. Tumour Biol, 2012, 33(1):183-194. https://doi.org/10.1007/s13277-0110261-7 PMID: 22081310

[26] de Vicente JC, Rodrigo JP, Rodriguez-Santamarta T, LequericaFernández P, Allonca E, García-Pedrero JM. Podoplanin expression in oral leukoplakia: tumorigenic role. Oral Oncol, 2013, 49(6):598-603. https://doi.org/10.1016/j.oraloncology. 2013.02.008 PMID: 23473850

[27] Kawaguchi H, El-Naggar AK, Papadimitrakopoulou V, Ren H, Fan YH, Feng L, Lee JJ, Kim E, Hong WK, Lippman SM, Mao L. Podoplanin: a novel marker for oral cancer risk in patients with oral premalignancy. J Clin Oncol, 2008, 26(3):354-360. https://doi.org/10.1200/JCO.2007.13.4072 PMID: 18202409

[28] Patil A, Patil K, Tupsakhare S, Gabhane M, Sonune S, Kandalgaonkar S. Evaluation of podoplanin in oral leukoplakia and oral squamous cell carcinoma. Scientifica (Cairo), 2015, 2015: 135298. https://doi.org/10.1155/2015/135298 PMID: 26558136 PMCID: PMC4618324

[29] A G D, Janardanan-Nair B, B R V. Podoplanin expression in oral potentially malignant disorders and oral squamous cell carcinoma. J Clin Exp Dent, 2017, 9(12):e1418-e1424. https:// doi.org/10.4317/jced.54213 PMID: 29410757 PMCID: PMC 5794119

[30] Margaritescu C, Raica M, Pirici D, Simionescu C, Mogoanta L, Stinga AC, Stinga AS, Ribatti D. Podoplanin expression in tumorfree resection margins of oral squamous cell carcinomas: an immunohistochemical and fractal analysis study. Histol Histopathol, 2010, 25(6):701-711. https://doi.org/10.14670/HH-25. 701 PMID: 20376776

[31] de Vicente JC, Santamarta TR, Rodrigo JP, García-Pedrero JM, Allonca E, Blanco-Lorenzo V. Expression of podoplanin in the invasion front of oral squamous cell carcinoma is not prognostic for survival. Virchows Arch, 2015, 466(5):549-558. https://doi.org/10.1007/s00428-015-1746-3 PMID: 25726183

[32] Bartuli FN, Luciani F, Caddeo F, Compagni S, Piva P, Ottria L, Arcuri C. Podoplanin in the development and progression of oral cavity cancer: a preliminary study. Oral Implantol (Rome), 2012, 5(2-3):33-41. PMID: 23285404 PMCID: PMC3505098

[33] Kreppel M, Scheer M, Drebber U, Ritter L, Zöller JE. Impact of podoplanin expression in oral squamous cell carcinoma: clinical and histopathologic correlations. Virchows Arch, 2010, 456(5):473-482. https://doi.org/10.1007/s00428-010-0915-7 PMID: 20393745 
[34] dos Santos Almeida A, Oliveira DT, Pereira MC, Faustino SES Nonogaki S, Carvalho AL, Kowalski LP. Podoplanin and VEGF$C$ immunoexpression in oral squamous cell carcinomas: prognostic significance. Anticancer Res, 2013, 33(9):39693976. PMID: 24023336

[35] Sharma G, Kumar R, Singh HP, Gupta M, Gupta M. Expression of podoplanin in tumor cells and lymphatic vessels in both tumoral and peritumoral areas and correlation with metastatic potential of oral squamous cell carcinoma. J Oral Maxillofac Pathol, 2021, 25(1):131-135. https://doi.org/10.4103/jomfp jomfp 481 20 PMID: 34349423 PMCID: PMC8272477

[36] Dong R, Zhang M, Hu Q, Zheng S, Soh A, Zheng Y, Yuan H. Galectin-3 as a novel biomarker for disease diagnosis and a target for therapy (Review). Int J Mol Med, 2018, 41(2):599_ 614. https://doi.org/10.3892/ijmm.2017.3311 PMID: 29207027 PMCID: PMC5752178

[37] Dumic J, Dabelic S, Flögel M. Galectin-3: an open-ended story. Biochim Biophys Acta, 2006, 1760(4):616-635. https:// doi.org/10.1016/j.bbagen.2005.12.020 PMID: 16478649

[38] Acikalin MF, Etiz D, Gurbuz MK, Ozudogru E, Canaz F, Colak E. Prognostic significance of galectin-3 and cyclin D1 expression in undifferentiated nasopharyngeal carcinoma. Med Oncol, 2012, 29(2):742-749. https://doi.org/10.1007/s12032-011-9 971-3 PMID: 21553103

[39] Aggarwal S, Sharma SC, Das SN. Galectin-1 and galectin-3: plausible tumour markers for oral squamous cell carcinoma and suitable targets for screening high-risk population. Clin Chim Acta, 2015, 442:13-21. https://doi.org/10.1016/j.cca.2014. 12.038 PMID: 25578395

[40] Lin Cl, Whang EE, Donner DB, Jiang X, Price BD, Carothers AM, Delaine T, Leffler H, Nilsson UJ, Nose V, Moore FD Jr, Ruan DT. Galectin-3 targeted therapy with a small molecule inhibitor activates apoptosis and enhances both chemosensitivity and radiosensitivity in papillary thyroid cancer. Mol Cancer Res, 2009, 7(10):1655-1662. https://doi.org/10.1158/1541-7786. MCR-09-0274 PMID: 19825987

[41] Honjo Y, Inohara H, Akahani S, Yoshii T, Takenaka Y, Yoshida J, Hattori K, Tomiyama Y, Raz A, Kubo T. Expression of cytoplasmic galectin-3 as a prognostic marker in tongue carcinoma. Clin Cancer Res, 2000, 6(12):4635-4640. PMID: 11156213

[42] Plzák J, Betka J, Smetana K Jr, Chovanec M, Kaltner H, André S, Kodet R, Gabius HJ. Galectin-3 - an emerging prognostic indicator in advanced head and neck carcinoma. Eur J Cancer, 2004, 40(15):2324-2330. https://doi.org/10.1016/ j.ejca.2004.06.025 PMID: 15454259

[43] Tokmak S, Arık D, Pınarbaşlı Ö, Gürbüz MK, Açıkalın MF. Evaluation and prognostic significance of galectin-3 expression in oral squamous cell carcinoma. Ear Nose Throat J, 2021, 100(5_Suppl):578S-583S. https://doi.org/10.1177/01455613 19893861 PMID: 31838918

[44] Wang LP, Chen SW, Zhuang SM, Li H, Song M. Galectin-3 accelerates the progression of oral tongue squamous cell carcinoma via a $\mathrm{Wnt} / \beta$-catenin-dependent pathway. Pathol Oncol Res, 2013, 19(3):461-474. https://doi.org/10.1007/s1 2253-013-9603-7 PMID: 23519607

[45] Weber M, Büttner-Herold M, Distel L, Ries J, Moebius $P$, Preidl R, Geppert Cl, Neukam FW, Wehrhan F. Galectin 3 expression in primary oral squamous cell carcinomas. BMC Cancer, 2017, 17(1):906. https://doi.org/10.1186/s12885-0173920-2 PMID: 29284429 PMCID: PMC5747141

[46] Alves PM, Godoy GP, Gomes DQ, Medeiros AMC, de Souza LB, da Silveira EJD, Vasconcelos MG, Queiroz LMG. Significance of galectins $-1,-3,-4$ and -7 in the progression of squamous cell carcinoma of the tongue. Pathol Res Pract, 2011, 207(4): 236-240. https://doi.org/10.1016/j.prp.2011.02.004 PMID: 21397408

[47] Wehrhan F, Büttner-Herold M, Distel L, Ries J, Moebius P, Preidl R, Geppert Cl, Neukam FW, Kesting M, Weber M. Galectin 3 expression in regional lymph nodes and lymph node metastases of oral squamous cell carcinomas. BMC Cancer, 2018, 18(1):823. https://doi.org/10.1186/s12885-0184726-6 PMID: 30115022 PMCID: PMC6097288

[48] Zou Z, Anisowicz A, Hendrix MJ, Thor A, Neveu M, Sheng S, Rafidi K, Seftor E, Sager R. Maspin, a serpin with tumorsuppressing activity in human mammary epithelial cells. Science, 1994, 263(5146):526-529. https://doi.org/10.1126/science.82 90962 PMID: 8290962
[49] Dzinic SH, Kaplun A, Li X, Bernardo M, Meng Y, Dean I, Krass D, Stemmer P, Shin N, Lonardo F, Sheng S. Identification of an intrinsic determinant critical for maspin subcellular localization and function. PLoS One, 2013, 8(11):e74502. https:// doi.org/10.1371/journal.pone.0074502 PMID: 24278104 PMCID: PMC3837015

[50] Lonardo F, Guan H, Dzinic S, Sheng S. Maspin expression patterns differ in the invasive versus lepidic growth pattern of pulmonary adenocarcinoma. Histopathology, 2014, 65(6):757763. https://doi.org/10.1111/his.12485 PMID: 25040445 PMCID: PMC4245330

[51] Pierson CR, McGowen R, Grignon D, Sakr W, Dey J, Sheng S. Maspin is up-regulated in premalignant prostate epithelia. Prostate, 2002, 53(4):255-262. https://doi.org/10.1002/pros. 10107 PMID: 12430137

[52] Wang Y, Sheng S, Zhang J, Dzinic S, Li S, Fang F, Wu N, Zheng $Q$, Yang Y. Elevated maspin expression is associated with better overall survival in esophageal squamous cell carcinoma (ESCC). PLoS One, 2013, 8(5):e63581. https:// doi.org/10.1371/journal.pone.0063581. Erratum in: PLoS One, 2014, 9(8):e104715. PMID: 23717449 PMCID: PMC3661574

[53] Shi HY, Zhang W, Liang R, Kittrell F, Templeton NS, Medina D, Zhang M. Modeling human breast cancer metastasis in mice: maspin as a paradigm. Histol Histopathol, 2003, 18(1):201-206. https://doi.org/10.14670/HH-18.201 PMID: 12507299

[54] Sheng $S$. The promise and challenge toward the clinical application of maspin in cancer. Front Biosci, 2004, 9:27332745. https://doi.org/10.2741/1432 PMID: 15353310

[55] lezzi G, Piattelli A, Rubini C, Goteri G, Artese L, Perrotti V, Carinci F. Maspin expression in oral squamous cell carcinoma. J Craniofac Surg, 2007, 18(5):1039-1043. https://doi.org/10. 1097/scs.0b013e31814b2a36 PMID: 17912078

[56] Yoshizawa K, Nozaki S, Kitahara H, Kato K, Noguchi N, Kawashiri S, Yamamoto E. Expression of urokinase-type plasminogen activator/urokinase-type plasminogen activator receptor and maspin in oral squamous cell carcinoma: association with mode of invasion and clinicopathological factors. Oncol Rep, 2011, 26(6):1555-1560. https://doi.org/ 10.3892/or.2011.1419 PMID: 21833477

[57] Yoshizawa K, Nozaki S, Okamune A, Kitahara H, Ohara T, Kato K, Kawashiri S, Yamamoto $\mathrm{E}$. Loss of maspin is a negative prognostic factor for invasion and metastasis in oral squamous cell carcinoma. J Oral Pathol Med, 2009, 38(6):535-539. https:// doi.org/10.1111/j.1600-0714.2009.00762.x PMID: 19473451

[58] Marioni G, Gaio E, Giacomelli L, Bertolin A, D'Alessandro E, Stramare R, Facco E, Staffieri A, Blandamura S. MASPIN subcellular localization and expression in oral cavity squamous cell carcinoma. Eur Arch Otorhinolaryngol, 2008, 265(Suppl 1): S97-S104. https://doi.org/10.1007/s00405-008-0583-2 PMID: 18236066

[59] Xia W, Lau YK, Hu MC, Li L, Johnston DA, Sheng Sj, ElNaggar A, Hung MC. High tumoral maspin expression is associated with improved survival of patients with oral squamous cell carcinoma. Oncogene, 2000, 19(20):2398-2403. https:// doi.org/10.1038/sj.onc.1203535 PMID: 10828881

[60] Yasumatsu R, Nakashima T, Hirakawa N, Kumamoto Y, Kuratomi $\mathrm{Y}$, Tomita K, Komiyama S. Maspin expression in stage I and II oral tongue squamous cell carcinoma. Head Neck, 2001, 23(11):962-966. https://doi.org/10.1002/hed.1139 PMID: 11754500

[61] Cho JH, Kim HS, Park CS, Kim JK, Jung KY, Shin BK, Kim HK. Maspin expression in early oral tongue cancer and its relation to expression of mutant-type p53 and vascular endothelial growth factor (VEGF). Oral Oncol, 2007, 43(3):272-277. https:// doi.org/10.1016/j.oraloncology.2006.03.018 PMID: 17174141

[62] Sakwe AM, Nguyen T, Athanasopoulos V, Shire K, Frappier L. Identification and characterization of a novel component of the human minichromosome maintenance complex. Mol Cell Biol, 2007, 27(8):3044-3055. https://doi.org/10.1128/MCB.02 384-06 PMID: 17296731 PMCID: PMC1899943

[63] Labib K, Kearsey SE, Diffley JF. MCM2-7 proteins are essential components of prereplicative complexes that accumulate cooperatively in the nucleus during $\mathrm{G} 1$-phase and are required to establish, but not maintain, the S-phase checkpoint. Mol Biol Cell, 2001, 12(11):3658-3667. https://doi.org/10.1091/mbc. 12.11.3658 PMID: 11694596 PMCID: PMC60283 
[64] Choy B, LaLonde A, Que J, Wu T, Zhou Z. MCM4 and MCM7, potential novel proliferation markers, significantly correlated with Ki-67, Bmi1, and cyclin E expression in esophageal adenocarcinoma, squamous cell carcinoma, and precancerous lesions. Hum Pathol, 2016, 57:126-135. https://doi.org/10.1016/j.hum path.2016.07.013 PMID: 27476776 PMCID: PMC5250507

[65] Kang W, Tong JHM, Chan AWH, Cheng ASL, Yu J, To K. MCM7 serves as a prognostic marker in diffuse-type gastric adenocarcinoma and siRNA-mediated knockdown suppresses its oncogenic function. Oncol Rep, 2014, 31(5):2071-2078. https://doi.org/10.3892/or.2014.3094 PMID: 24647462

[66] Valverde LF, de Freitas RD, Pereira TA, de Resende MF Agra IMG, Dos Santos JN, Dos Reis MG, Sales CBS, Gurgel Rocha CA. MCM3: a novel proliferation marker in oral squamous cell carcinoma. Appl Immunohistochem Mol Morphol, 2018, 26(2):120-125. https://doi.org/10.1097/PAI.0000000000000 397 PMID: 27258565

[67] Feng CJ, Li HJ, Li JN, Lu YJ, Liao GQ. Expression of Mcm7 and Cdc6 in oral squamous cell carcinoma and precancerous lesions. Anticancer Res, 2008, 28(6A):3763-3769. PMID: 19189662

[68] Tamura T, Shomori K, Haruki T, Nosaka K, Hamamoto Y, Shiomi T, Ryoke K, Ito H. Minichromosome maintenance-7 and geminin are reliable prognostic markers in patients with oral squamous cell carcinoma: immunohistochemical study. J Oral Pathol Med, 2010, 39(4):328-334. https://doi.org/10. 1111/j.1600-0714.2009.00861.x PMID: 20136698

\section{Corresponding authors}

Claudiu Mărgăritescu, Professor, MD, PhD, Department of Pathology, Faculty of Dentistry, University of Medicine and Pharmacy of Craiova, 661 May Avenue, 200628 Craiova, Dolj County, Romania; Phone +40740-152 550, e-mail: c_margaritescu2000@yahoo.com

Daniela Dumitrescu, Associate Professor, MD, PhD, Department of Radiology and Medical Imaging, University of Medicine and Pharmacy of Craiova, 1 Tabaci Street, 200642 Craiova, Dolj County, Romania; Phone/Fax +40251502 263, e-mail: daniela.dumitrescu@gmail.com

Received: May 25, 2021

Accepted: September 15, 2021 